\title{
Efficient Nucleophilic Aromatic Substitution between Aryl Nitrofluorides and Alkynes
}

Patrick L. DeRoy, Simon Surprenant, Megan

Bertrand-Laperle, and Christiane Yoakim*

Department of Chemistry, Boehringer Ingelheim (Canada) Ltd, Research and Development, 2100 Cunard Street, Laval, Quebec, Canada H7S 2 G5

cyoakim@lav.boehringer-ingelheim.com

Table of Content

General

Typical Procedure

Spectral data for compound in Table 1, entry 1

Spectral data for compound in Table 2, entry 2

Spectral data for compound in Table 2, entry 3

Spectral data for compound in Table 2, entry 4

Spectral data for compound in Table 2, entry 5

Spectral data for compound in Table 2, entry 6

Spectral data for compound in Table 2, entry 7

Spectral data for compound in Table 2, entry 8

Spectral data for compound in Table 2, entry 9 
Spectral data for compound in Table 2, entry 12

${ }^{13} \mathrm{H}-\mathrm{NMR}$ for compound in Table 1 , entry 1

${ }^{13} \mathrm{H}-\mathrm{NMR}$ for compound in Table 2, entry 2

${ }^{13} \mathrm{H}$-NMR for compound in Table 2, entry 3

${ }^{1}$ H-NMR for compound in Table 2, entry 5

${ }^{13} \mathrm{H}$-NMR for compound in Table 2, entry $6 \quad$ S22

${ }^{1} \mathrm{H}-\mathrm{NMR}$ for compound in Table 2, entry $7 \quad$ S23 
${ }^{13} \mathrm{H}-\mathrm{NMR}$ for compound in Table 2, entry 7

${ }^{1}$ H-NMR for compound in Table 2, entry 8 S25

${ }^{13} \mathrm{H}$-NMR for compound in Table 2, entry 8 S26

${ }^{1}$ H-NMR for compound in Table 2, entry 9 S27

${ }^{13} \mathrm{H}$-NMR for compound in Table 2, entry 9 S28

${ }^{1}$ H-NMR for compound in Table 2, entry 10 S29

${ }^{13} \mathrm{H}$-NMR for compound in Table 2, entry 10 S30

${ }^{1}$ H-NMR for compound in Table 2, entry 11 S31

${ }^{13} \mathrm{H}$-NMR for compound in Table 2, entry 11 S32

${ }^{1}$ H-NMR for compound in Table 2, entry 12 S33

${ }^{13} \mathrm{H}$-NMR for compound in Table 2, entry 12 S34

${ }^{1} \mathrm{H}$-NMR for compound in Table 2, entry 13 S35

${ }^{13} \mathrm{H}$-NMR for compound in Table 2, entry 13 S36

${ }^{1} \mathrm{H}$-NMR for compound in Table 2, entry 14 S37

${ }^{13}$ H-NMR for compound in Table 2, entry 14 S38

${ }^{1} \mathrm{H}$-NMR for compound in Table 2, entry 15 S39

${ }^{13}$ H-NMR for compound in Table 2, entry 15 S40

${ }^{1}$ H-NMR for compound in Table 2, entry 16 S41

${ }^{13} \mathrm{H}$-NMR for compound in Table 2, entry 16 S42

${ }^{1}$ H-NMR for compound in Table 3 , entry 1 S43

${ }^{13}$ H-NMR for compound in Table 3, entry 1 S44

${ }^{1}$ H-NMR for compound in Table 3, entry 2 S45

${ }^{13} \mathrm{H}$-NMR for compound in Table 3, entry 2 
${ }^{1}$ H-NMR for compound in Table 3, entry 3

${ }^{13} \mathrm{H}-\mathrm{NMR}$ for compound in Table 3, entry $3 \quad$ S48

${ }^{1} \mathrm{H}$-NMR for compound in Table 3, entry 4

${ }^{13} \mathrm{H}$-NMR for compound in Table 3, entry $4 \quad$ S50

${ }^{1} \mathrm{H}$-NMR for compound in Table 3, entry 5

${ }^{13} \mathrm{H}-\mathrm{NMR}$ for compound in Table 3, entry 5 
General: All reactions were run under inert atmosphere using anhydrous solvents. Unless otherwise noted, materials were obtained from commercial sources and used without further purification. Reactions were followed by HPLC and TLC. The purity of each inhibitor was determined by high-performance liquid chromatography (HPLC) and was $>95 \%$ unless otherwise noted. ${ }^{1} \mathrm{H}$ NMR and ${ }^{13} \mathrm{C}$ NMR spectra were obtained on a Bruker AMX 400 spectrometer. ${ }^{1} \mathrm{H}$ assignment abbreviations are the following: singlet (s), doublet (d), triplet (t), quartet (q), pentet (p), broad singlet (bs), broad multiplet (bm), broad quartet (bq), doublet of doublets (dd), doublet of triplets (dt), and multiplet (m). Electrospray mass spectrum (ES-MS) were recorded on a Micromass-Quattro-II mass spectrometer. FTIR spectra were taken on a Perkin-Elmer 241 polarimeter. Purification was performed using flash column chromatography (FCC) on silica gel $(10-40 \mu \mathrm{m}$ or 230-400 mesh, Silicycle).

Typical Procedure: A rt solution of nitro-fluoroaryl (1.0 eq) and alkyne (1.1 eq) in THF $(0.3 \mathrm{M})$ was treated dropwise with sodium hexamethyldisilazane (NaHMDS, $1.0 \mathrm{M}$ in THF, $1.5 \mathrm{eq}$ ) then stirred for $1-5 \mathrm{~h}$ at $60^{\circ} \mathrm{C}$. On completion of the reaction, the mixture was diluted with $\mathrm{Et}_{2} \mathrm{O}$, washed with water and brine, dried over $\mathrm{MgSO}_{4}$, filtered, concentrated and purified by flash chromatography using $\mathrm{Et}_{2} \mathrm{O} /$ hexanes or EtOAc/hexanes to afford the desired product.

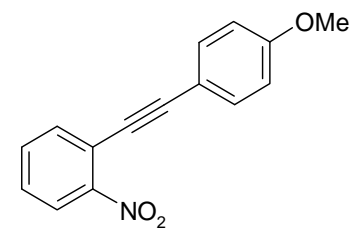

1-[(4-methoxyphenyl)ethynyl]-2-nitrobenzene (Table 1, entry 1): orange solid; $\mathrm{R}_{f}=0.21\left(20 \% \mathrm{Et}_{2} \mathrm{O} /\right.$ hexanes $) ;{ }^{1} \mathrm{H}$ NMR $\left(\mathrm{CDCl}_{3}\right.$, $400 \mathrm{MHz}) \delta 8.09(\mathrm{~d}, J=8.2 \mathrm{~Hz}, 1 \mathrm{H}), 7.71(\mathrm{~d}, J=7.8 \mathrm{~Hz}, 1 \mathrm{H}), 7.60$ $(\mathrm{t}, J=7.6 \mathrm{~Hz}, 1 \mathrm{H}) ; 7.56(\mathrm{~d}, J=8.6 \mathrm{~Hz}, 2 \mathrm{H}), 7.44(\mathrm{t}, J=7.8 \mathrm{~Hz}$, $1 \mathrm{H}), 7.92(\mathrm{~d}, J=8.4 \mathrm{~Hz}, 2 \mathrm{H}), 3.86(\mathrm{~s}, 3 \mathrm{H}) ;{ }^{13} \mathrm{C} \mathrm{NMR}\left(\mathrm{CDCl}_{3}, 100\right.$ MHz) $\delta 160.6,149.6,134.5,133.9,133.0,128.2,124.9,119.4,114.6,114.3,97.8,84.1$, 55.6; MS (EI, M+•) 253; HRMS for $\mathrm{C}_{15} \mathrm{H}_{11} \mathrm{NO}_{3}$ calc 253.0739, found 253.0732; FTIR $3001,2214,1601,1567,1523,1332,1287,1136,1026,835,684 \mathrm{~cm}^{-1}$.

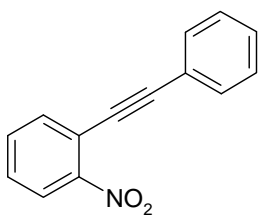

1-nitro-2-(phenylethynyl)benzene (Table 2, entry 1): All data are consistent with literature: Li, J.-H.; Zhang, X.-D., Xie, Y.-X. Eur. J. Org. Chem. 2005, 4256.

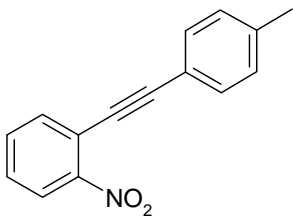

1-[(4-methylphenyl)ethynyl]-2-nitrobenzene (Table 2, entry 2): orange solid; $\mathrm{R}_{f}=0.37\left(20 \% \mathrm{Et}_{2} \mathrm{O} /\right.$ hexanes $) ;{ }^{1} \mathrm{H}$ NMR $\left(\mathrm{CDCl}_{3}, 400\right.$ MHz) $\delta 8.09(\mathrm{~d}, J=8.2 \mathrm{~Hz}, 1 \mathrm{H}), 7.72(\mathrm{~d}, J=7.8 \mathrm{~Hz}, 1 \mathrm{H}), 7.60(\mathrm{t}, J=$ $7.4 \mathrm{~Hz}, 1 \mathrm{H}), 7.50$ (d, $J=8.0 \mathrm{~Hz}, 2 \mathrm{H}), 7.46$ (t, $J=8.2 \mathrm{~Hz}, 1 \mathrm{H}), 7.20$ $(\mathrm{d}, J=7.6 \mathrm{~Hz}, 2 \mathrm{H}), 2.39(\mathrm{~s}, 3 \mathrm{H}) ;{ }^{13} \mathrm{C}$ NMR $\left(\mathrm{CDCl}_{3}, 100 \mathrm{MHz}\right) \delta$ $149.5,139.6,134.5,132.7,131.9,129.2,128.3,124.7,119.3,119.0,97.5,84.3,21.6$; MS (EI, M+•) 237; HRMS for $\mathrm{C}_{15} \mathrm{H}_{11} \mathrm{NO}_{2}$ calc 237.0790, found 237.0786; FTIR 2218, 1604, $1524,1342,1291,817,744 \mathrm{~cm}^{-1}$. 


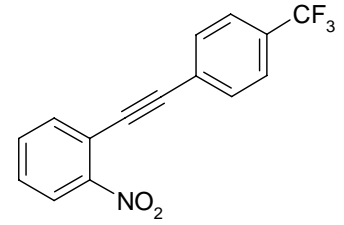

1-nitro-2-\{[4-(trifluoromethyl)phenyl]ethynyl\}benzene (Table 2, Entry 3): yellow solid; $\mathrm{R}_{f}=0.34\left(20 \% \mathrm{Et}_{2} \mathrm{O} /\right.$ hexanes $) ;{ }^{1} \mathrm{H}$ NMR $\left(\mathrm{CDCl}_{3}, 400 \mathrm{MHz}\right) \delta 8.14(\mathrm{~d}, J=8.2 \mathrm{~Hz}, 1 \mathrm{H}), 7.78(\mathrm{t}, J=8.3 \mathrm{~Hz}$, 2H), $7.73(\mathrm{~d}, J=7.6 \mathrm{~Hz}, 1 \mathrm{H}), 7.65(\mathrm{t}, J=7.6 \mathrm{~Hz}, 1 \mathrm{H}), 7.58(\mathrm{t}, J=$ 7.6 Hz, $1 \mathrm{H}), 7.52(\mathrm{~m}, 2 \mathrm{H}) ;{ }^{13} \mathrm{C} \mathrm{NMR}\left(\mathrm{CDCl}_{3}, 100 \mathrm{MHz}\right) \delta 149.2$, 135.0, 134.5, 133.0, 131.6, 129.2, 129.0, 126.0, 124.7, 122.1, 120.6, 118.2, 92.6, 89.9; MS (EI, M+•) 291; HRMS for $\mathrm{C}_{15} \mathrm{H}_{8} \mathrm{NO}_{2} \mathrm{~F}_{3}$ calc 291.0507, found 291.0500.

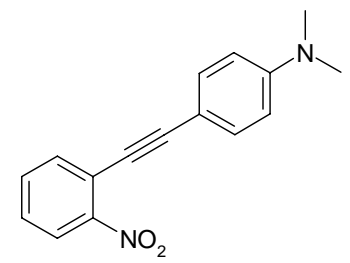

$\mathbf{N}, \boldsymbol{N}$-dimethyl-4-[(2-nitrophenyl)ethynyl]aniline (Table 2, entry 4): red solid; $\mathrm{R}_{f}=0.18\left(20 \% \mathrm{Et}_{2} \mathrm{O} /\right.$ hexanes $) ;{ }^{1} \mathrm{H} \mathrm{NMR}\left(\mathrm{CDCl}_{3}, 400\right.$ $\mathrm{MHz}) \delta 8.07(\mathrm{~d}, J=8.2 \mathrm{~Hz}, 1 \mathrm{H}), 7.68(\mathrm{~d}, J=7.8 \mathrm{~Hz}, 1 \mathrm{H}), 7.56(\mathrm{t}$, $J=7.6 \mathrm{~Hz}, 1 \mathrm{H}), 7.49$ (d, $J=8.4 \mathrm{~Hz}, 2 \mathrm{H}), 7.38(\mathrm{t}, J=7.8 \mathrm{~Hz}, 1 \mathrm{H})$, $6.67(\mathrm{~d}, J=8.4 \mathrm{~Hz}, 2 \mathrm{H}), 3.03(\mathrm{~s}, 6 \mathrm{H}) ;{ }^{13} \mathrm{C} \mathrm{NMR}\left(\mathrm{CDCl}_{3}, 100\right.$ $\mathrm{MHz}) \delta 150.7,148.9,134.1,133.4,132.6,127.3,124.7,119.9$, 111.6, 108.7, 99.5, 83.7, 40.1; MS (EI, M+•) 266; HRMS for $\mathrm{C}_{16} \mathrm{H}_{14} \mathrm{~N}_{2} \mathrm{O}_{2}$ calc 266.1055, found 266.1048; FTIR 2904, 2209, 1600, 1519, 1339, 1228, 821, $740 \mathrm{~cm}^{-1}$.

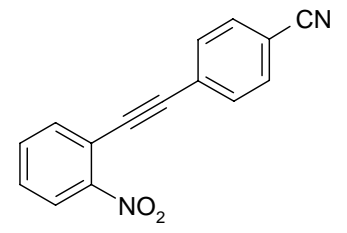

4-[(2-nitrophenyl)ethynyl]benzonitrile (Table 2, entry 5): yellow solid; $\mathrm{R}_{f}=0.13\left(20 \% \mathrm{Et}_{2} \mathrm{O} /\right.$ hexanes $) ;{ }^{1} \mathrm{H} \mathrm{NMR}\left(\mathrm{CDCl}_{3}, 400\right.$ $\mathrm{MHz}) \delta 8.15(\mathrm{~d}, J=8.2 \mathrm{~Hz}, 1 \mathrm{H}), 7.75(\mathrm{~d}, J=7.8 \mathrm{~Hz}, 1 \mathrm{H}), 7.69$ (s, $4 \mathrm{H}), 7.66(\mathrm{t}, J=7.6 \mathrm{~Hz}, 1 \mathrm{H}), 7.55(\mathrm{t}, J=8.0 \mathrm{~Hz}, 1 \mathrm{H}) ;{ }^{13} \mathrm{C} \mathrm{NMR}$ $\left(\mathrm{CDCl}_{3}, 100 \mathrm{MHz}\right) \delta 149.6,134.7,133.0,132.4,132.3,129.5$, 127.2, 124.9, 118.3, 117.7, 112.4, 94.7, 88.7; MS (EI, M+•) 248; HRMS for $\mathrm{C}_{15} \mathrm{H}_{8} \mathrm{~N}_{2} \mathrm{O}_{2}$ calc 248.0586, found 248.0589; FTIR 2225, 1607, 1567, 1522, 1339, 1295, 909, 837, $743,554 \mathrm{~cm}^{-1}$.

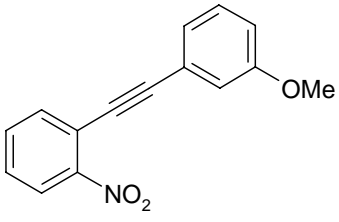

1-[(3-methoxyphenyl)ethynyl]-2-nitrobenzene (Table 2, entry 6): orange oil; $\mathrm{R}_{f}=0.24\left(20 \% \mathrm{Et}_{2} \mathrm{O} /\right.$ hexanes $) ;{ }^{1} \mathrm{H} \mathrm{NMR}\left(\mathrm{CDCl}_{3}\right.$, $400 \mathrm{MHz}) \delta 8.10(\mathrm{~d}, J=8.2 \mathrm{~Hz}, 1 \mathrm{H}), 7.74(\mathrm{~d}, J=7.8 \mathrm{~Hz}, 1 \mathrm{H})$, $7.62(\mathrm{t}, J=7.6 \mathrm{~Hz}, 1 \mathrm{H}), 7.48(\mathrm{t}, J=8.1 \mathrm{~Hz}, 1 \mathrm{H}), 7.30(\mathrm{t}, J=8.2$ $\mathrm{Hz}, 1 \mathrm{H}), 7.21(\mathrm{~d} J=7.4 \mathrm{~Hz}, 1 \mathrm{H}), 7.12(\mathrm{~s}, 1 \mathrm{H}), 6.96(\mathrm{~d}, J=8.2 \mathrm{~Hz}$, $1 \mathrm{H}), 3.85(\mathrm{~s}, 3 \mathrm{H}) ;{ }^{13} \mathrm{C} \mathrm{NMR}\left(\mathrm{CDCl}_{3}, 100 \mathrm{MHz}\right) \delta 159.4,149.6,134.6,132.8,129.5$, $128.6,124.7,124.6,123.3,118.7,116.5,116.0$, 97.0, 84.5, 55.3; MS (EI, M+•) 253; HRMS for $\mathrm{C}_{15} \mathrm{H}_{11} \mathrm{NO}_{3}$ calc 253.0379, found 253.0732; FTIR 2210, 1608, 1573, 1524, $1491,1342,1226,1043,783,744,685 \mathrm{~cm}^{-1}$.

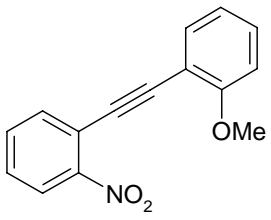

1-methoxy-2-[(2-nitrophenyl)ethynyl]benzene (Table 2, entry 7): yellow solid; $\mathrm{R}_{f}=0.19\left(20 \% \mathrm{Et}_{2} \mathrm{O} /\right.$ hexanes $) ;{ }^{1} \mathrm{H} \mathrm{NMR}\left(\mathrm{CDCl}_{3}, 400\right.$ 
MHz) $\delta 8.10(\mathrm{~d}, J=8.2 \mathrm{~Hz}, 1 \mathrm{H}), 7.77(\mathrm{~d}, J=7.6 \mathrm{~Hz}, 1 \mathrm{H}), 7.59(\mathrm{~m}, 2 \mathrm{H}), 7.46(\mathrm{t}, J=8.2$ $\mathrm{Hz}, 1 \mathrm{H}), 7.37(\mathrm{t}, J=7.6 \mathrm{~Hz}, 1 \mathrm{H}), 6.98(\mathrm{t}, J=7.4 \mathrm{~Hz}, 1 \mathrm{H}), 6.94,(\mathrm{~d}, J=7.4 \mathrm{~Hz}, 1 \mathrm{H}), 3.96$ $(\mathrm{s}, 3 \mathrm{H}) ;{ }^{13} \mathrm{C}$ NMR $\left(\mathrm{CDCl}_{3}, 100 \mathrm{MHz}\right) \delta 160.4,149.3,134.7,134.0,132.7,130.8,128.3$, 124.6, 120.5, 119.1, 111.6, 110.8, 93.9, 88.7, 55.9; MS (EI, M+•) 253; HRMS for $\mathrm{C}_{15} \mathrm{H}_{11} \mathrm{NO}_{3}$ calc 253.0739, found 253.0732; FTIR 2215, 1607, 1565, 1517, 1461, 1338, $1250,1022,746 \mathrm{~cm}^{-1}$.

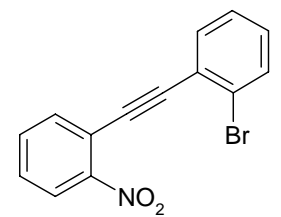

1-bromo-2-[(2-nitrophenyl)ethynyl]benzene (Table 2, entry 8): yellow solid; $\mathrm{R}_{f}=0.32$ (10\% EtOAc/hexanes); ${ }^{1} \mathrm{H} \mathrm{NMR}\left(\mathrm{CDCl}_{3}, 400\right.$ $\mathrm{MHz}) \delta 8.11(\mathrm{~d}, J=7.4 \mathrm{~Hz}, 1 \mathrm{H}), 7.80(\mathrm{dd}, J=7.6,1.2 \mathrm{~Hz}, 1 \mathrm{H}), 7.62$ $(\mathrm{m}, 3 \mathrm{H}), 7.50(\mathrm{dt}, J=7.8,1.4 \mathrm{~Hz}, 1 \mathrm{H}), 7.34(\mathrm{dt}, J=7.5,0.8 \mathrm{~Hz}, 1 \mathrm{H})$, $7.24(\mathrm{dt} J=7.8,1.8,1 \mathrm{H}) ;{ }^{13} \mathrm{C} \mathrm{NMR}\left(\mathrm{CDCl}_{3}, 100 \mathrm{MHz}\right) \delta 149.2,134.9$, 134.0, 132.9, 132.5, 130.3, 128.9, 127.1, 125.7, 124.7, 124.6, 118.4, 95.4, 88.9; MS (EI, $\mathrm{M}+\bullet$ ) 301; HRMS for $\mathrm{C}_{14} \mathrm{H}_{8} \mathrm{NO}_{2} \mathrm{Br}$ calc 300.9738, found 300.9744 .

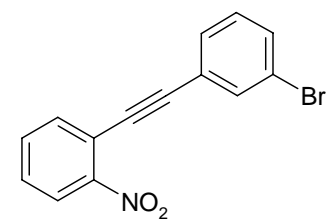

1-[(3-bromophenyl)ethynyl]-2-nitrobenzene (Table 2, entry 9): yellow solid; $\mathrm{R}_{f}=0.25\left(20 \% \mathrm{Et}_{2} \mathrm{O} /\right.$ hexanes $) ;{ }^{1} \mathrm{H} \mathrm{NMR}\left(\mathrm{CDCl}_{3}, 400\right.$ $\mathrm{MHz}) \delta 8.12(\mathrm{~d}, J=8.3 \mathrm{~Hz}, 1 \mathrm{H}), 7.74(\mathrm{~m}, 2 \mathrm{H}), 7.64(\mathrm{t}, J=7.6 \mathrm{~Hz}$, $1 \mathrm{H}), 7.53(\mathrm{~m}, 3 \mathrm{H}), 7.27(\mathrm{~m}, 1 \mathrm{H}) ;{ }^{13} \mathrm{C} \mathrm{NMR}\left(\mathrm{CDCl}_{3}, 100 \mathrm{MHz}\right) \delta$ $149.6,134.59,134.57,132.9,132.3,130.5,129.9,128.9,124.8$, 124.3, 122.2, 118.2, 95.2, 85.9; MS (EI, M+•) 301, 303; HRMS for $\mathrm{C}_{14} \mathrm{H}_{8} \mathrm{NO}_{2} \mathrm{Br}$ calc 300.9738 , found 300.9744; FTIR 2223, 1608, 1524, 1343, 881, 782, 743, $678 \mathrm{~cm}^{-1}$.

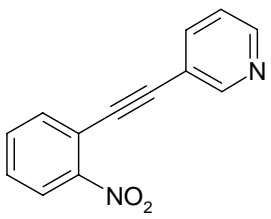

3-[(2-nitrophenyl)ethynyl]pyridine (Table 2, entry 10): yellow solid; $\mathrm{R}_{f}=0.13\left(50 \% \mathrm{Et}_{2} \mathrm{O} /\right.$ hexanes $) ;{ }^{1} \mathrm{H} \mathrm{NMR}\left(\mathrm{CDCl}_{3}, 400 \mathrm{MHz}\right) \delta$ $8.84(\mathrm{~s}, 1 \mathrm{H}), 8.62(\mathrm{dd}, J=4.9,1.8 \mathrm{~Hz}, 1 \mathrm{H}), 8.14(\mathrm{~d}, J=8.2 \mathrm{~Hz}, 1 \mathrm{H})$, $7.89(\mathrm{~m}, 1 \mathrm{H}), 7.74(\mathrm{~d}, J=7.6 \mathrm{~Hz}, 1 \mathrm{H}), 7.65(\mathrm{t}, J=7.65 \mathrm{~Hz}, 1 \mathrm{H}), 7.53$ $(\mathrm{m}, 1 \mathrm{H}), 7.33(\mathrm{~m}, 1 \mathrm{H}) ;{ }^{13} \mathrm{C} \mathrm{NMR}\left(\mathrm{CDCl}_{3}, 100 \mathrm{MHz}\right) \delta 152.4,149.5$, 149.4, 138.8, 134.6, 133.0, 129.1, 124.8, 123.1, 119.6, 118.0, 93.3, 87.8; MS (EI, M+•) 224; HRMS for $\mathrm{C}_{13} \mathrm{H}_{8} \mathrm{NO}_{2}$ calc 224.0586, found 224.0591; FTIR 2222, 1520, 1484 , $1340,788,744,700 \mathrm{~cm}^{-1}$.

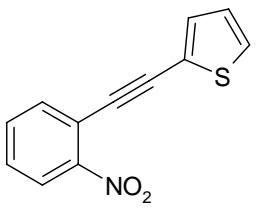

2-[(2-nitrophenyl)ethynyl]thiophene (Table 2, entry 11): red oil; $\mathrm{R}_{f}$ $=0.29(20 \% \mathrm{EtOAc} /$ hexanes $) ;{ }^{1} \mathrm{H} \mathrm{NMR}\left(\mathrm{CDCl}_{3}, 400 \mathrm{MHz}\right) \delta 8.12(\mathrm{~d}, J$ $=8.2 \mathrm{~Hz}, 1 \mathrm{H}), 7.71(\mathrm{~d}, J=6.8 \mathrm{~Hz}, 1 \mathrm{H}), 7.62(\mathrm{t}, J=7.6 \mathrm{~Hz}, 1 \mathrm{H}), 7.47(\mathrm{t}$, $J=8.2 \mathrm{~Hz}, 1 \mathrm{H}), 7.41(\mathrm{~m}, 2 \mathrm{H}), 7.07(\mathrm{dd}, J=5.1,3.9 \mathrm{~Hz}, 1 \mathrm{H}) ;{ }^{13} \mathrm{C} \mathrm{NMR}$ $\left(\mathrm{CDCl}_{3}, 100 \mathrm{MHz}\right) \delta 148.9,134.2,133.4,132.9,128.9,128.5,127.3$, 124.8, 122.2, 118.5, 90.5, 88.8; MS (EI, M+•) 229; HRMS for $\mathrm{C}_{12} \mathrm{H}_{7} \mathrm{NO}_{2} \mathrm{~S}$ calc 229.0197, found 229.0202; FTIR 3106, 2203, 1607, 1524, 1342, 1216, 852, 743, $706 \mathrm{~cm}^{-1}$. 


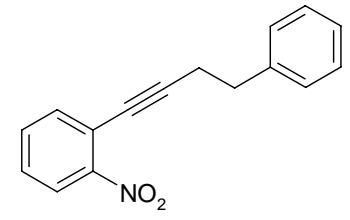

1-nitro-2-(4-phenylbut-1-yn-1-yl)benzene (Table 2, entry 12): red oil; $\mathrm{R}_{f}=0.24\left(20 \% \mathrm{Et}_{2} \mathrm{O} /\right.$ hexanes $) ;{ }^{1} \mathrm{H}$ NMR $\left(\mathrm{CDCl}_{3}, 400\right.$ MHz) $\delta 7.95(\mathrm{~d}, J=8.0 \mathrm{~Hz}, 1 \mathrm{H}), 7.49(\mathrm{~m}, 2 \mathrm{H}), 7.37(\mathrm{~m}, 1 \mathrm{H}), 7.32-$ $7.19(\mathrm{~m}, 5 \mathrm{H}), 2.94(\mathrm{t}, J=7.5 \mathrm{~Hz}, 2 \mathrm{H}), 2.76(\mathrm{t}, J=7.5 \mathrm{~Hz}, 2 \mathrm{H}) ;{ }^{13} \mathrm{C}$ NMR $\left(\mathrm{CDCl}_{3}, 100 \mathrm{MHz}\right) \delta 150.0,140.3,134.8,132.5,128.5$, 128.4, 128.0, 126.4, 124.4, 119.1, 98.3, 76.6, 34.6, 22.0; MS (EI, M+•) 251; HRMS for $\mathrm{C}_{16} \mathrm{H}_{13} \mathrm{NO}_{2}$ calc 251.0946, found 251.0950; FTIR 2929, 2229, 1607, 1526, 1344, 849, $784,745,699 \mathrm{~cm}^{-1}$.

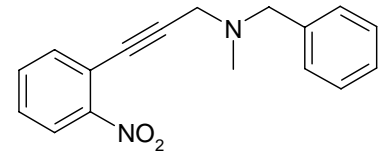

$N$-benzyl- $N$-methyl-3-(2-nitrophenyl)prop-2-yn-1-amine (Table 2, entry 13): red oil; $\mathrm{R}_{f}=0.17$ (20\% EtOAc/hexanes); ${ }^{1} \mathrm{H}$ $\operatorname{NMR}\left(\mathrm{CDCl}_{3}, 400 \mathrm{MHz}\right) \delta 8.05(\mathrm{~d}, J=8.2 \mathrm{~Hz}, 1 \mathrm{H}), 7.66(\mathrm{~d}, J=$ $7.4 \mathrm{~Hz}, 1 \mathrm{H}), 7.58(\mathrm{t}, J=7.6 \mathrm{~Hz}, 1 \mathrm{H}), 7.46(\mathrm{t}, J=7.8 \mathrm{~Hz}, 1 \mathrm{H})$, $7.35(\mathrm{~m}, 5 \mathrm{H}), 3.70(\mathrm{~s}, 2 \mathrm{H}), 3.60(\mathrm{~s}, 2 \mathrm{H}), 2.46(\mathrm{~s}, 3 \mathrm{H}) ;{ }^{13} \mathrm{C} \mathrm{NMR}\left(\mathrm{CDCl}_{3}, 100 \mathrm{MHz}\right) \delta$ $149.8,138.3,134.9,132.7,129.2,128.4,128.3,127.2,124.4,118.5,93.4,80.9,60.0$, 45.7, 42.0; MS (EI, M+•) 280; HRMS for $\mathrm{C}_{17} \mathrm{H}_{16} \mathrm{~N}_{2} \mathrm{O}_{2}$ calc 280.1212, found 280.1207; FTIR 2794, 1608, 1526, 1344, 1026, 852, 743, $699 \mathrm{~cm}^{-1}$.

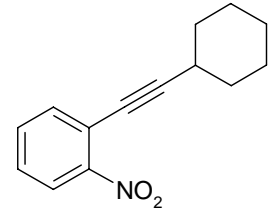

1-(cyclohexylethynyl)-2-nitrobenzene (Table 2, entry 14): yellow oil; $\mathrm{R}_{f}=0.42\left(20 \% \mathrm{Et}_{2} \mathrm{O} /\right.$ hexanes $) ;{ }^{1} \mathrm{H} \mathrm{NMR}\left(\mathrm{CDCl}_{3}, 400 \mathrm{MHz}\right) \delta 7.98$ $(\mathrm{d}, J=8.2 \mathrm{~Hz}, 1 \mathrm{H}), 7.59(\mathrm{~m}, 1 \mathrm{H}), 7.52(\mathrm{t}, J=7.5 \mathrm{~Hz}, 1 \mathrm{H}), 7.39(\mathrm{t}, J=$ $7.39 \mathrm{~Hz}, 1 \mathrm{H}), 2.69(\mathrm{~m}, 1 \mathrm{H}), 1.89(\mathrm{~m}, 2 \mathrm{H}), 1.78(\mathrm{~m}, 2 \mathrm{H}), 1.62(\mathrm{~m}, 2 \mathrm{H})$, $1.54(\mathrm{~m}, 1 \mathrm{H}), 1.39(\mathrm{~m}, 3 \mathrm{H}) ;{ }^{13} \mathrm{C} \mathrm{NMR}\left(\mathrm{CDCl}_{3}, 100 \mathrm{MHz}\right) \delta 150.1$, 134.7, 132.4, 127.7, 124.3, 119.4, 103.2, 76.0, 32.1, 29.9, 25.8, 24.6; MS (EI, M+•) 229; HRMS for $\mathrm{C}_{14} \mathrm{H}_{15} \mathrm{NO}_{2}$ calc 229.1103, found 229.1098; FTIR 2931, 2855, 2230, 1607 , $1526,1449,1346,856,783,745 \mathrm{~cm}^{-1}$.

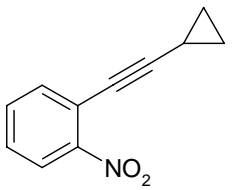

1-(cyclopropylethynyl)-2-nitrobenzene (Table 2, entry 15): yellow oil; $\mathrm{R}_{f}=0.35\left(20 \% \mathrm{Et}_{2} \mathrm{O} /\right.$ hexanes $) ;{ }^{1} \mathrm{H} \mathrm{NMR}\left(\mathrm{CDCl}_{3}, 400 \mathrm{MHz}\right) \delta 7.98$ $(\mathrm{d}, J=8.0 \mathrm{~Hz}, 1 \mathrm{H}), 7.55(\mathrm{dt}, J=7.6,1.4 \mathrm{~Hz}, 1 \mathrm{H}), 7.51(\mathrm{dt}, J=8.0,1.0$ $\mathrm{Hz}, 1 \mathrm{H}), 7.38(\mathrm{dt}, J=8.4,1.6 \mathrm{~Hz}, 1 \mathrm{H}), 1.55(\mathrm{~m}, 1 \mathrm{H}), 0.94(\mathrm{~m}, 4 \mathrm{H}) ;{ }^{13} \mathrm{C}$ $\mathrm{NMR}\left(\mathrm{CDCl}_{3}, 100 \mathrm{MHz}\right) \delta 149.9,134.6,132.5,127.6,124.4,119.4$, 102.7, 9.1, 0.6; MS (EI, M+•) 187; HRMS for $\mathrm{C}_{11} \mathrm{H}_{9} \mathrm{NO}_{2}$ calc 187.0633, found 187.0624; FTIR 2231, 1607, 1525, 1344, 955, 858, $745 \mathrm{~cm}^{-1}$.

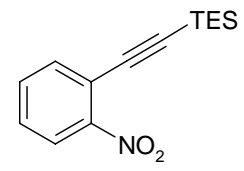

Triethyl-(2-nitro-phenylethynyl)-silane (Table 2, entry 16): yellow oil; $\mathrm{R}_{f}=0.38\left(20 \% \mathrm{Et}_{2} \mathrm{O} /\right.$ hexanes $) ;{ }^{1} \mathrm{H} \mathrm{NMR}\left(\mathrm{CDCl}_{3}, 400 \mathrm{MHz}\right) \delta 8.02(\mathrm{~d}, J=$ $8.2 \mathrm{~Hz}, 1 \mathrm{H}), 7.68(\mathrm{~d}, J=7.6 \mathrm{~Hz}, 1 \mathrm{H}), 7.56(\mathrm{t}, J=7.6 \mathrm{~Hz} ; 1 \mathrm{H}), 7.45(\mathrm{t}, J=$ $7.8 \mathrm{~Hz}, 1 \mathrm{H}), 1.07(\mathrm{t}, J=7.8 \mathrm{~Hz}, 9 \mathrm{H}), 0.72(\mathrm{q}, J=7.8 \mathrm{~Hz}, 6 \mathrm{H}) ;{ }^{13} \mathrm{C}$ NMR $\left(\mathrm{CDCl}_{3}, 100 \mathrm{MHz}\right) \delta 150.1,153.3,132.6,128.7,124.4,118.5,101.6,100.4,7.4,4.2 ; \mathrm{MS}$ (ESI, Pos) 262.1; MS (EI, M+•) 261; HRMS for $\mathrm{C}_{14} \mathrm{H}_{19} \mathrm{NO}_{2} \mathrm{Si}$ calc 261.1185, found 261.1180; FTIR 2956, 2912, 2876, 1606, 1530, 1346, 1008, 865, 820, $727 \mathrm{~cm}^{-1}$. 


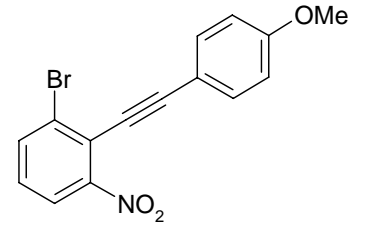

1-bromo-2-[(4-methoxyphenyl)ethynyl]-3-nitrobenzene (Table 3, entry 1): orange solid; $\mathrm{R}_{f}=0.17\left(20 \% \mathrm{Et}_{2} \mathrm{O} /\right.$ hexanes $) ;{ }^{1} \mathrm{H}$ NMR $\left(\mathrm{CDCl}_{3}, 400 \mathrm{MHz}\right) \delta 7.98(\mathrm{~d}, J=8.2 \mathrm{~Hz}, 1 \mathrm{H}), 7.90(\mathrm{~d}, J=8.0 \mathrm{~Hz}$, $1 \mathrm{H}), 7.60(\mathrm{~d}, J=8.8 \mathrm{~Hz}, 2 \mathrm{H}), 7.28(\mathrm{t}, J=8.1 \mathrm{~Hz}, 1 \mathrm{H}), 6.93(\mathrm{~d}, J=$ $8.8 \mathrm{~Hz}, 2), 3.86(\mathrm{~s}, 3 \mathrm{H}) ;{ }^{13} \mathrm{C}$ NMR $\left(\mathrm{CDCl}_{3}, 100 \mathrm{MHz}\right) \delta 160.8$, $150.8,136.7,133.8,128.0,127.9,123.4,120.9,114.2,114.1,103.3,82.9,55.4$; MS (EI, $\mathrm{M}+\bullet$ ) 331; HRMS for $\mathrm{C}_{15} \mathrm{H}_{10} \mathrm{NO}_{3} \mathrm{Br}$ calc 330.9844, found 330.9838; FTIR 2213, 1589, $1510,1247,1145,825,740 \mathrm{~cm}^{-1}$.

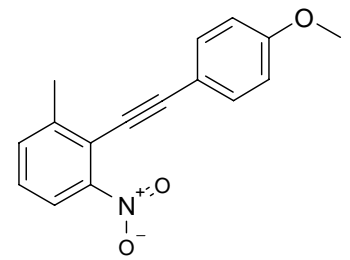

2-[(4-methoxyphenyl)ethynyl]-1-methyl-3-nitrobenzene (Table 3, entry 2): orange solid; $\mathrm{R}_{f}=0.19$ (10\% EtOAc/hexanes); ${ }^{1} \mathrm{H}$ NMR $\left(\mathrm{CDCl}_{3}, 400 \mathrm{MHz}\right) \delta 7.86(\mathrm{~d}, J=8.0 \mathrm{~Hz}, 1 \mathrm{H}), 7.55(\mathrm{~d}, J=$ $8.6 \mathrm{~Hz}, 2 \mathrm{H}), 7.50(\mathrm{~d}, J=7.7 \mathrm{~Hz}, 1 \mathrm{H}), 7.31(\mathrm{t}, J=8.0 \mathrm{~Hz}, 1 \mathrm{H}), 6.91$ $(\mathrm{d}, J=8.6 \mathrm{~Hz}, 2 \mathrm{H}), 3.86(\mathrm{~s}, 3 \mathrm{H}), 2.62(\mathrm{~s}, 3 \mathrm{H}) ;{ }^{13} \mathrm{C} \mathrm{NMR}\left(\mathrm{CDCl}_{3}\right.$, $100 \mathrm{MHz}) \delta 160.4,150.2,142.8,133.7,133.5,127.2,122.0,118.5$, 114.6, 114.1, 102.2, 82.2, 55.3, 21.3; MS (EI, M+•) 267; HRMS for $\mathrm{C}_{16} \mathrm{H}_{13} \mathrm{NO}_{3}$ calc 267.0895, found 267.0888; FTIR 2837, 2209, 1600, 1524, 1510, 1336, 1287, 1251, 1165, $1030,828,802,748 \mathrm{~cm}^{-1}$.

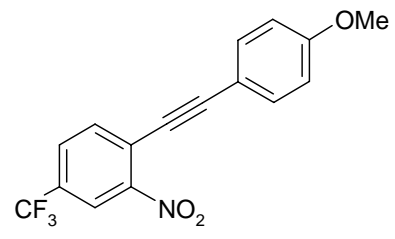

1-[(4-methoxyphenyl)ethynyl]-2-nitro-4-(trifluoromethyl)benzene (Table 3, entry 3): orange solid; $R_{f}=0.24(10 \%$ EtOAc/hexanes); ${ }^{1} \mathrm{H}$ NMR $\left(\mathrm{CDCl}_{3}, 400 \mathrm{MHz}\right) \delta 8.36(\mathrm{~s}, 1 \mathrm{H})$, $7.83(\mathrm{~s}, 2 \mathrm{H}), 7.58(\mathrm{~d}, J=8.8 \mathrm{~Hz}, 2 \mathrm{H}), 6.93(\mathrm{~d}, J=8.8 \mathrm{~Hz}, 2 \mathrm{H})$, $3.87(\mathrm{~s}, 3 \mathrm{H}) ;{ }^{13} \mathrm{C} \mathrm{NMR}\left(\mathrm{CDCl}_{3}, 100 \mathrm{MHz}\right) \delta 160.9,149.0,135.0$, 134.0, 129.17, 122.2, 122.1, 114.2, 113.6, 101.1, 83.3, 55.4 ; MS (EI, M+•) 321; HRMS for $\mathrm{C}_{16} \mathrm{H}_{10} \mathrm{NO}_{3} \mathrm{~F}_{3}$ calc 321.0613, found 321.0609; FTIR (neat) 2210, 1605, 1536, 1326, $1250,1121,1090,829 \mathrm{~cm}^{-1}$.

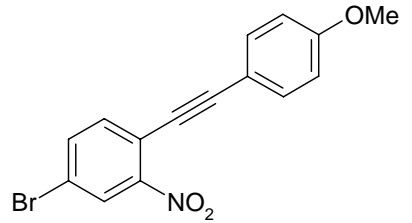

4-[(4-bromo-2-nitrophenyl)ethynyl]phenyl methyl ether (Table 3, entry 4): orange solid; $\mathrm{R}_{f}=0.26\left(20 \% \mathrm{Et}_{2} \mathrm{O} /\right.$ hexanes); ${ }^{1} \mathrm{H}$ NMR $\left(\mathrm{CDCl}_{3}, 400 \mathrm{MHz}\right) \delta 8.24(\mathrm{~d}, J=2.0 \mathrm{~Hz}, 1 \mathrm{H}), 7.72$ (dd, $J=8.3,2.0 \mathrm{~Hz}, 1 \mathrm{H}), 7.56(\mathrm{~d}, J=8.3 \mathrm{~Hz}, 1 \mathrm{H}), 7.55(\mathrm{~d}, J=$ $8.8 \mathrm{~Hz}, 2 \mathrm{H}), 6.91(\mathrm{~d}, J=8.8 \mathrm{~Hz}, 2 \mathrm{H}), 3.86(\mathrm{~s}, 3 \mathrm{H}) ;{ }^{13} \mathrm{C}$ NMR $\left(\mathrm{CDCl}_{3}, 100 \mathrm{MHz}\right) \delta 160.6,149.5,135.9,135.3,133.7,127.8,121.2,118.2,114.2,114.1$, 99.0, 83.2, 55.3; MS (EI, M+•) 331, 333; HRMS for $\mathrm{C}_{15} \mathrm{H}_{11} \mathrm{NO}_{2}$ calc 330.9844, found 330.9838; FTIR 2967, 2216, 1603, 1549, 1517, 1340, 1249, 1140, 1031, $827 \mathrm{~cm}^{-1}$.

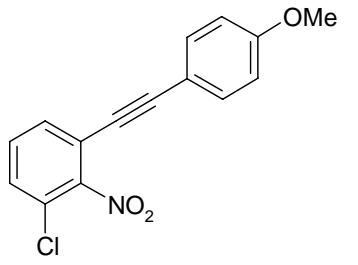

1-chloro-3-[(4-methoxyphenyl)ethynyl]-2-nitrobenzene (Table 3, entry 5): off-white solid; $\mathrm{R}_{f}=0.17\left(20 \% \mathrm{Et}_{2} \mathrm{O} /\right.$ hexanes $) ;{ }^{1} \mathrm{H}$ 
NMR $\left(\mathrm{CDCl}_{3}, 400 \mathrm{MHz}\right) \delta 7.53(\mathrm{dd}, J=7.4,1.0 \mathrm{~Hz}, 1 \mathrm{H}), 7.46(\mathrm{~d}, J=8.6 \mathrm{~Hz}, 2 \mathrm{H}), 7.42$ $(\mathrm{m}, 2 \mathrm{H}), 6.89(\mathrm{~d}, J=8.8 \mathrm{~Hz}, 1 \mathrm{H}), 3.85(\mathrm{~s}, 3 \mathrm{H}) ;{ }^{13} \mathrm{C} \mathrm{NMR}\left(\mathrm{CDCl}_{3}, 100 \mathrm{MHz}\right) \delta 160.6$, 133.6, 130.9, 130.6, 129.6, $125.3118 .8,114.1,113.4,97.4,80.5,55.3$; MS (EI, M+•) 287; HRMS for $\mathrm{C}_{15} \mathrm{H}_{10} \mathrm{NO}_{3} \mathrm{Cl}$ calc 287.0349, found 287.0345; FTIR 2958, 2836, 2232, $2203,1602,1590,1543,1508,1371,1292,1251,1165,1029,831,792 \mathrm{~cm}^{-1}$. 


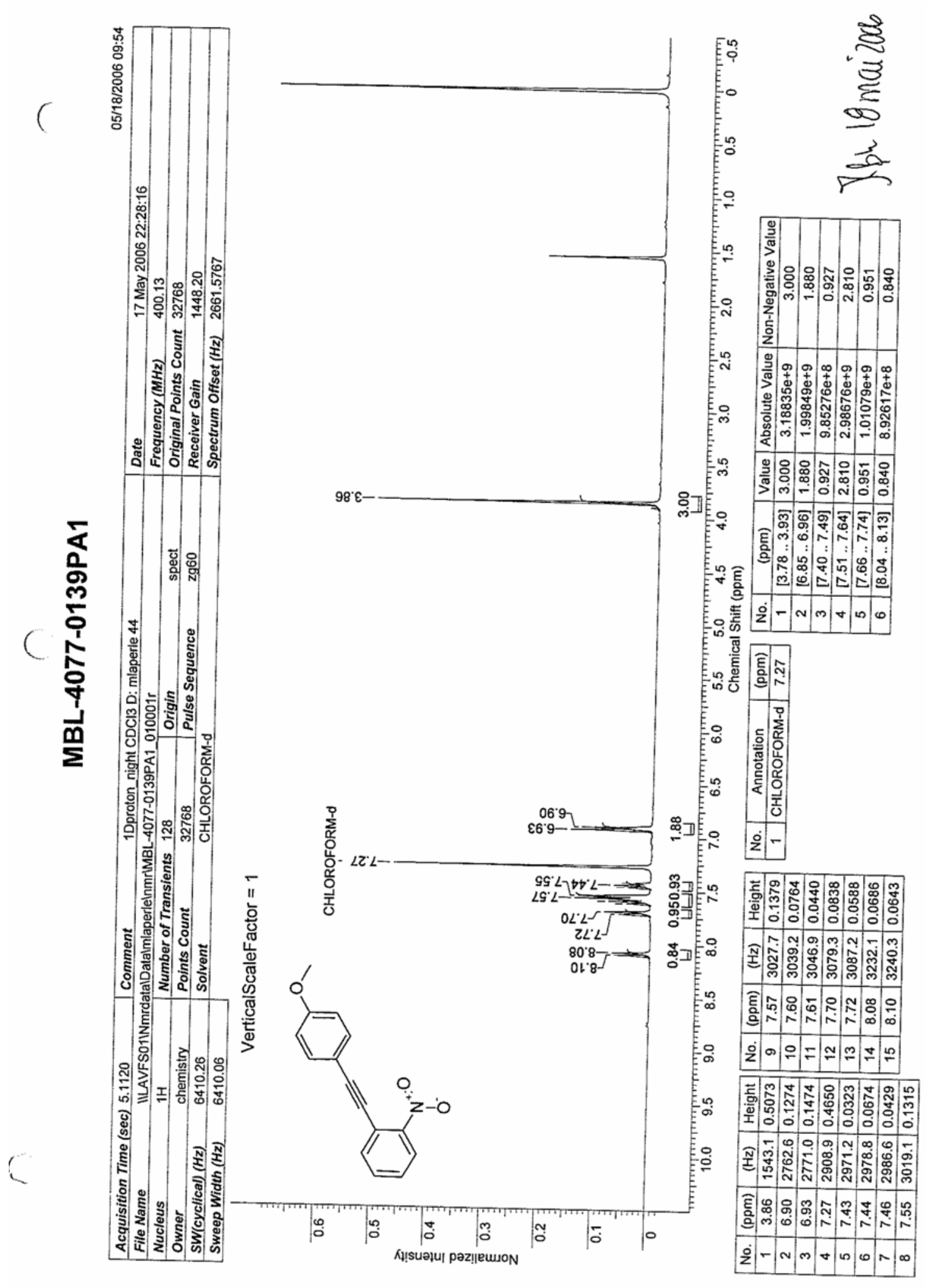




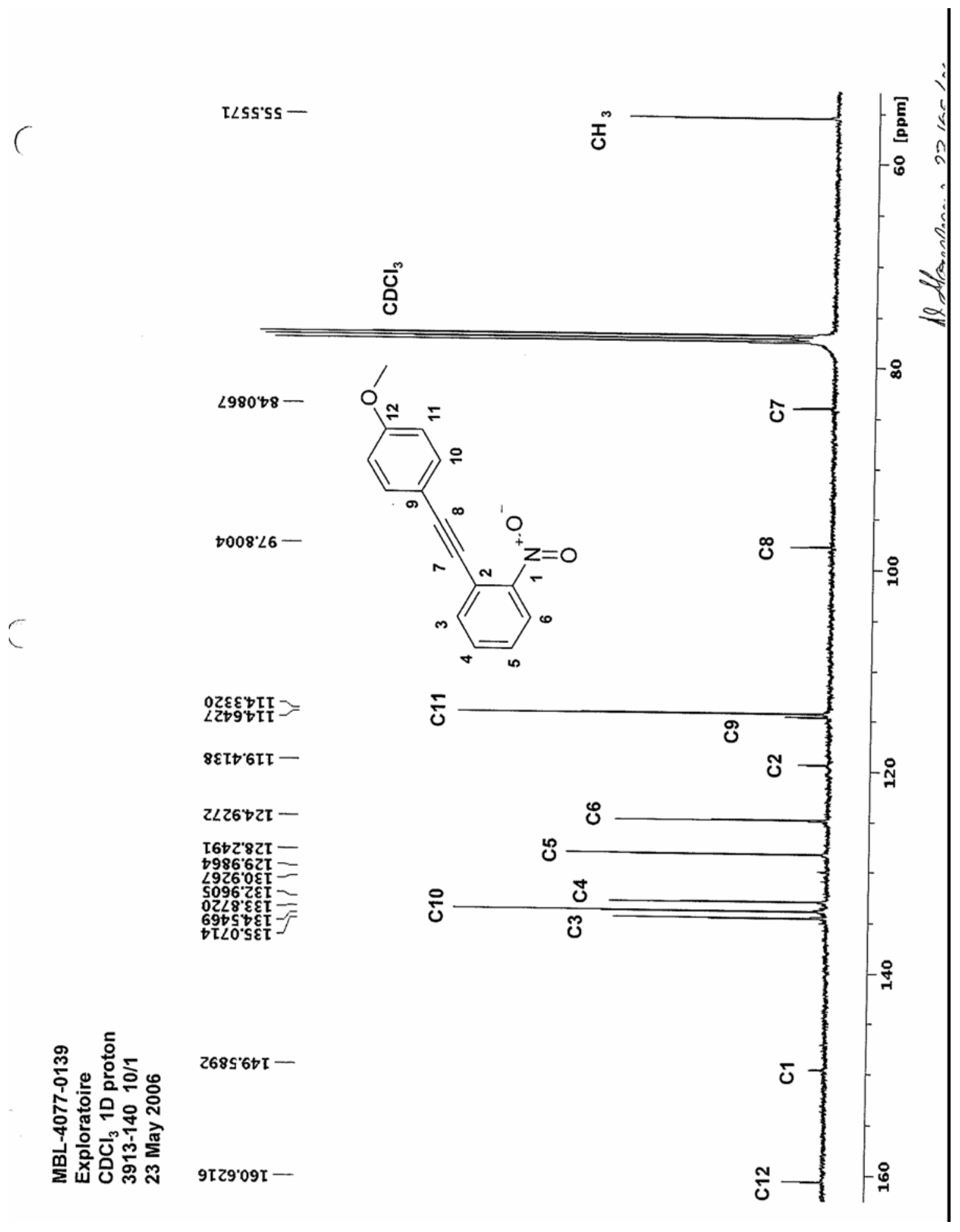




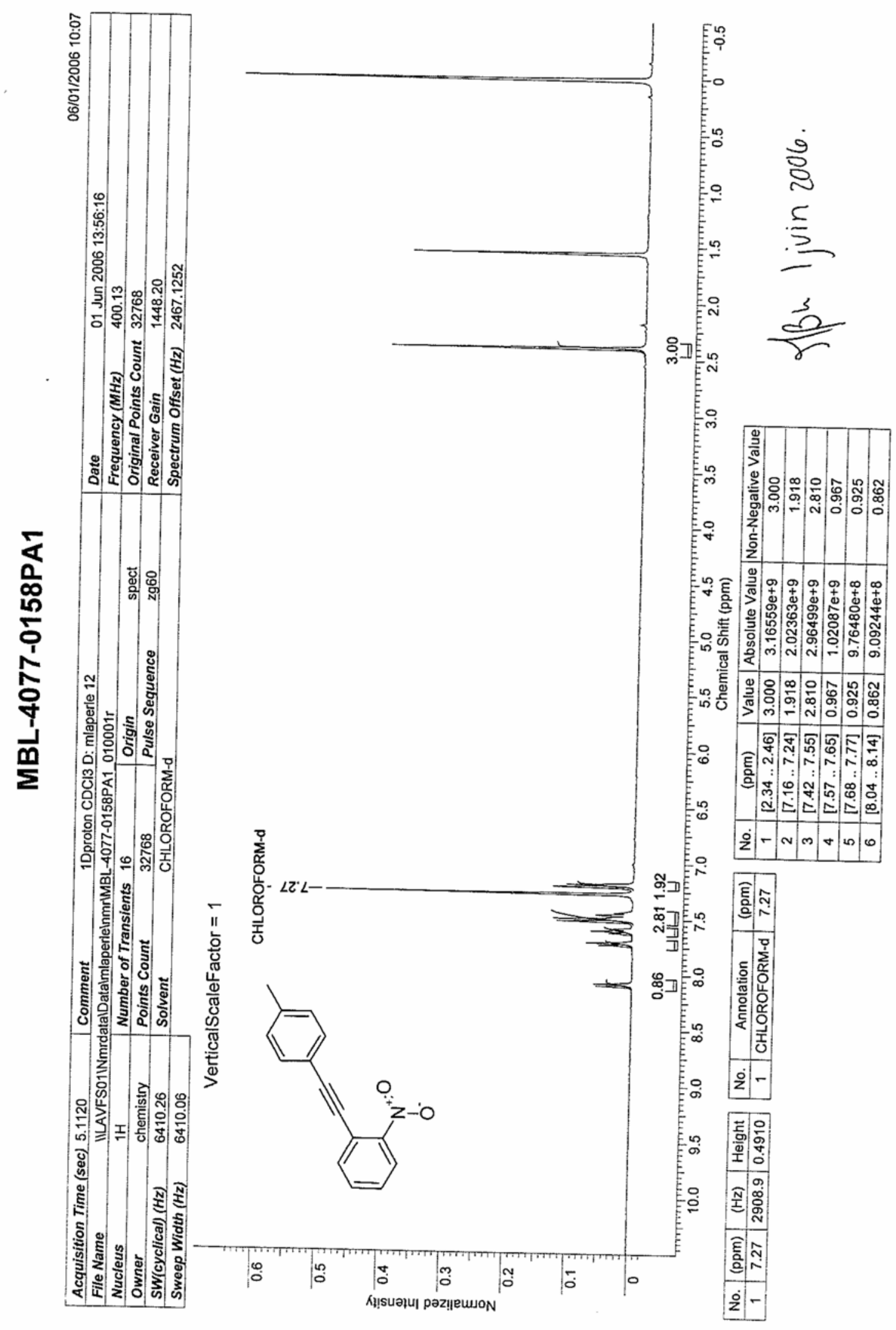




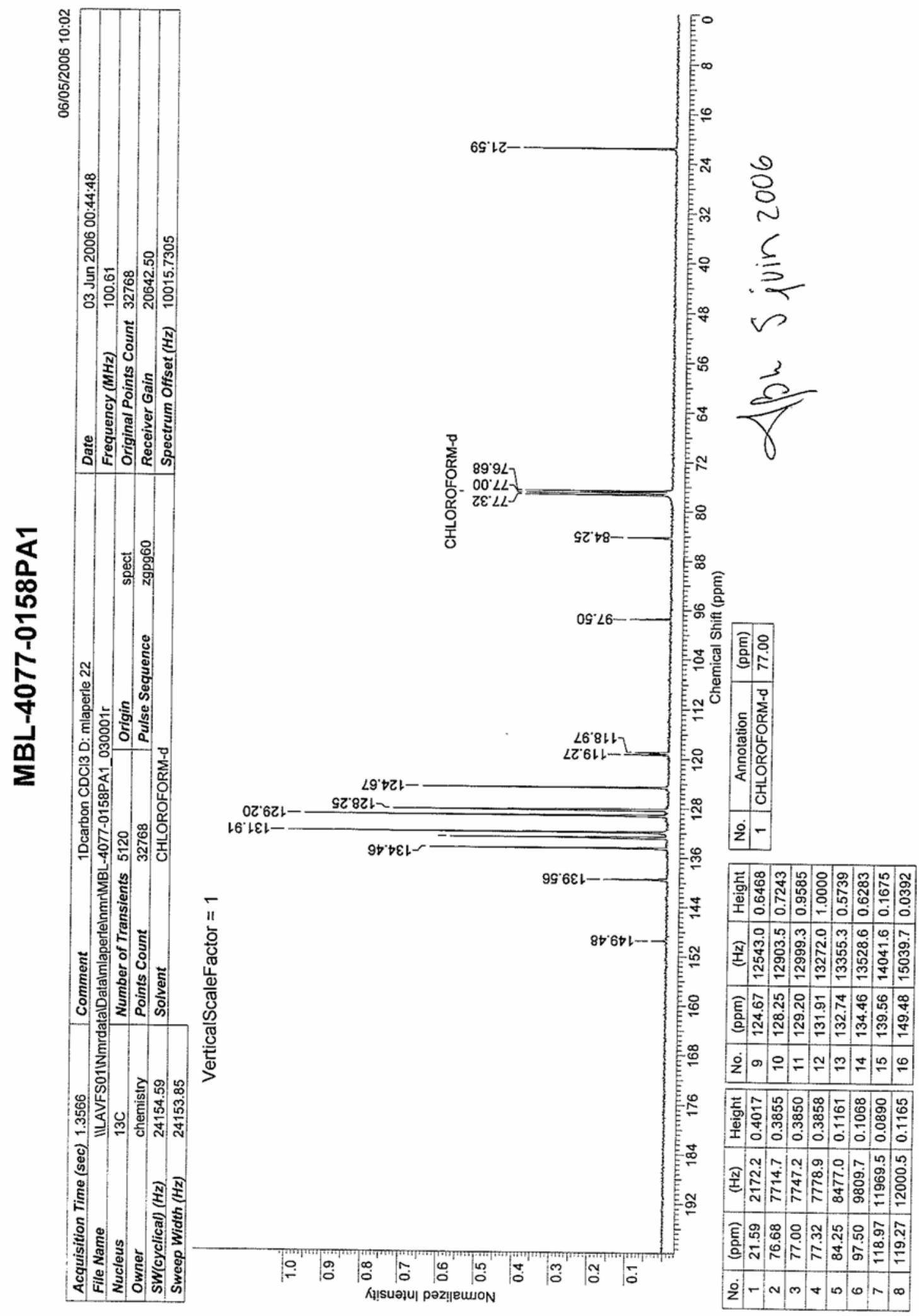




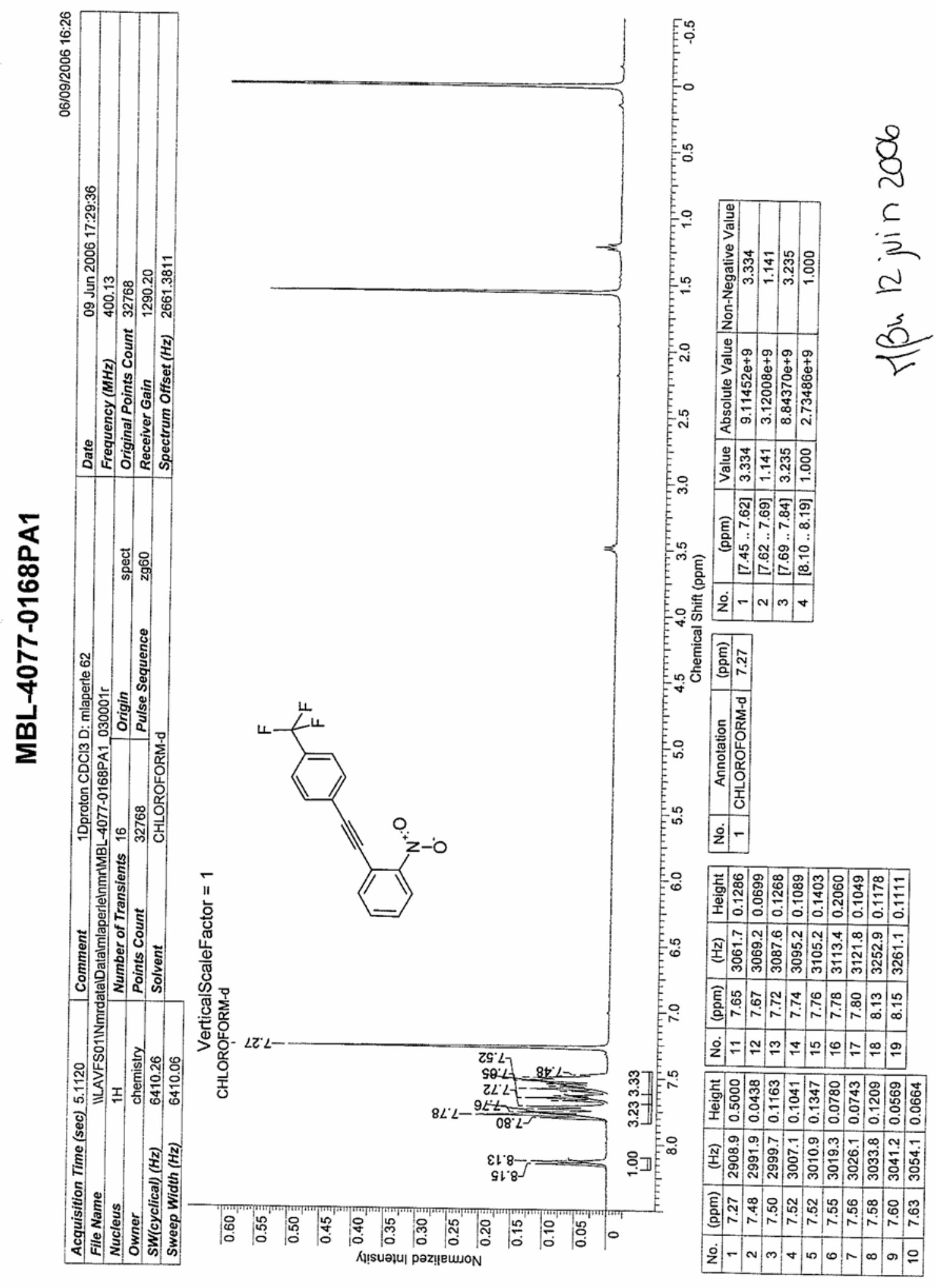




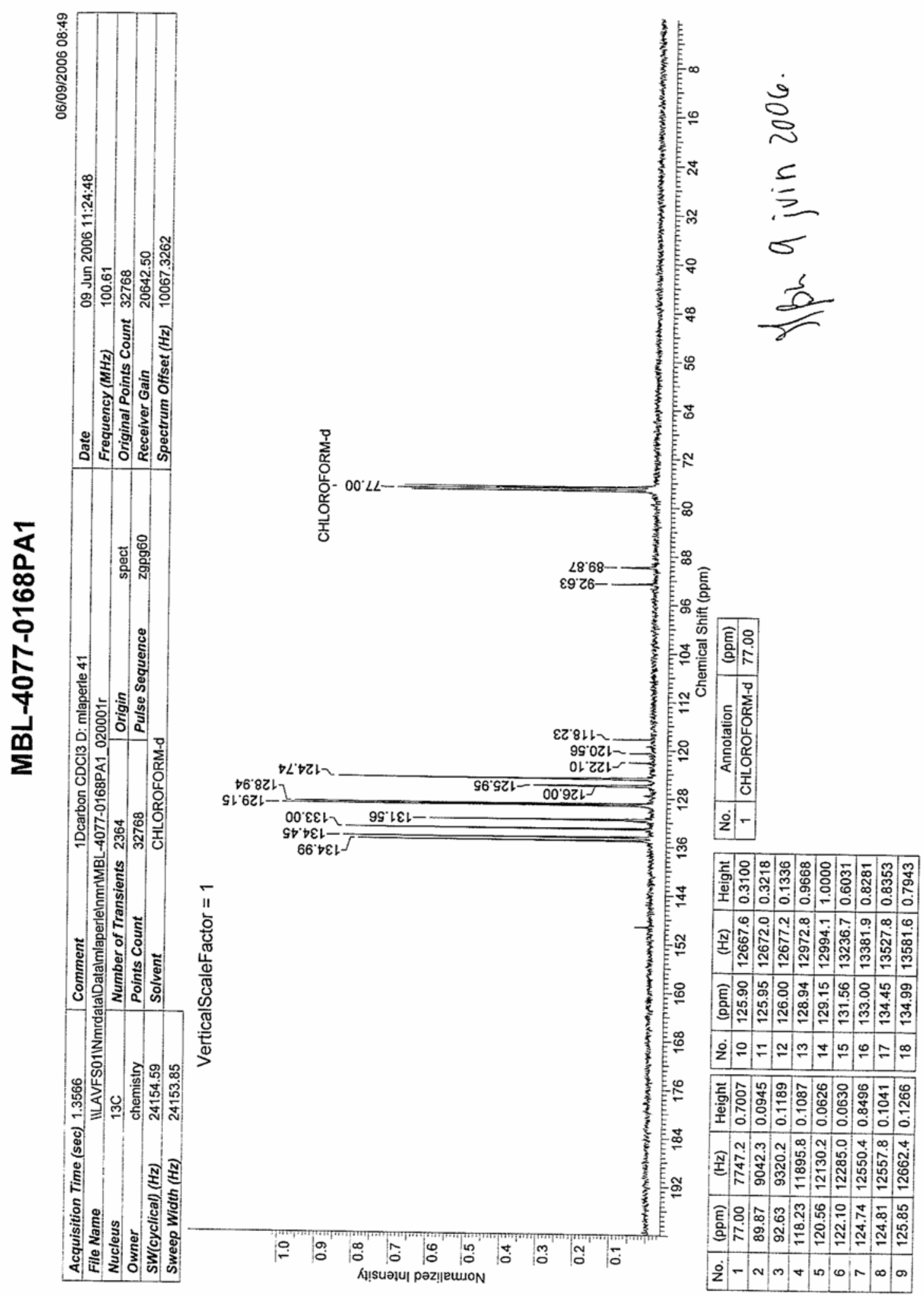




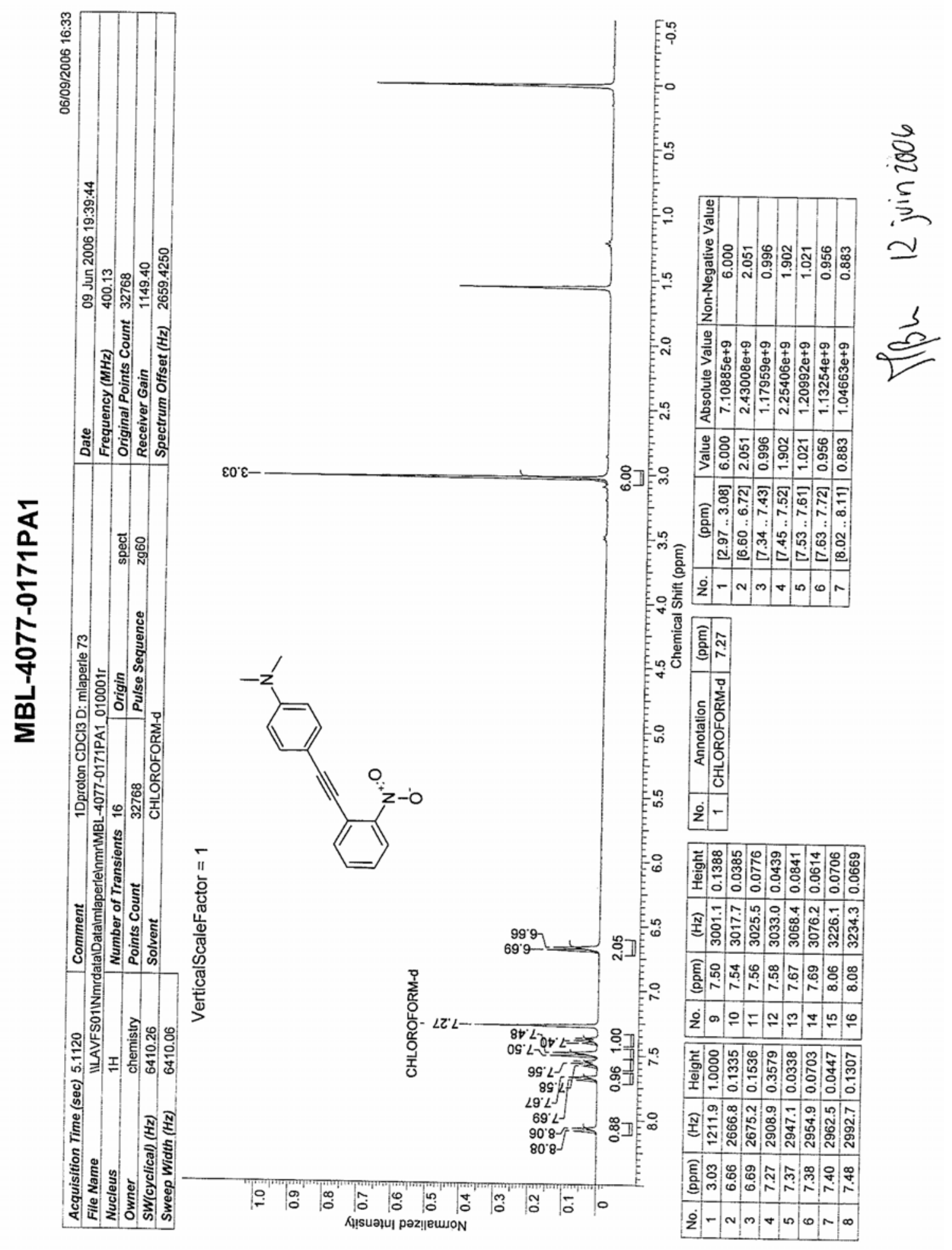




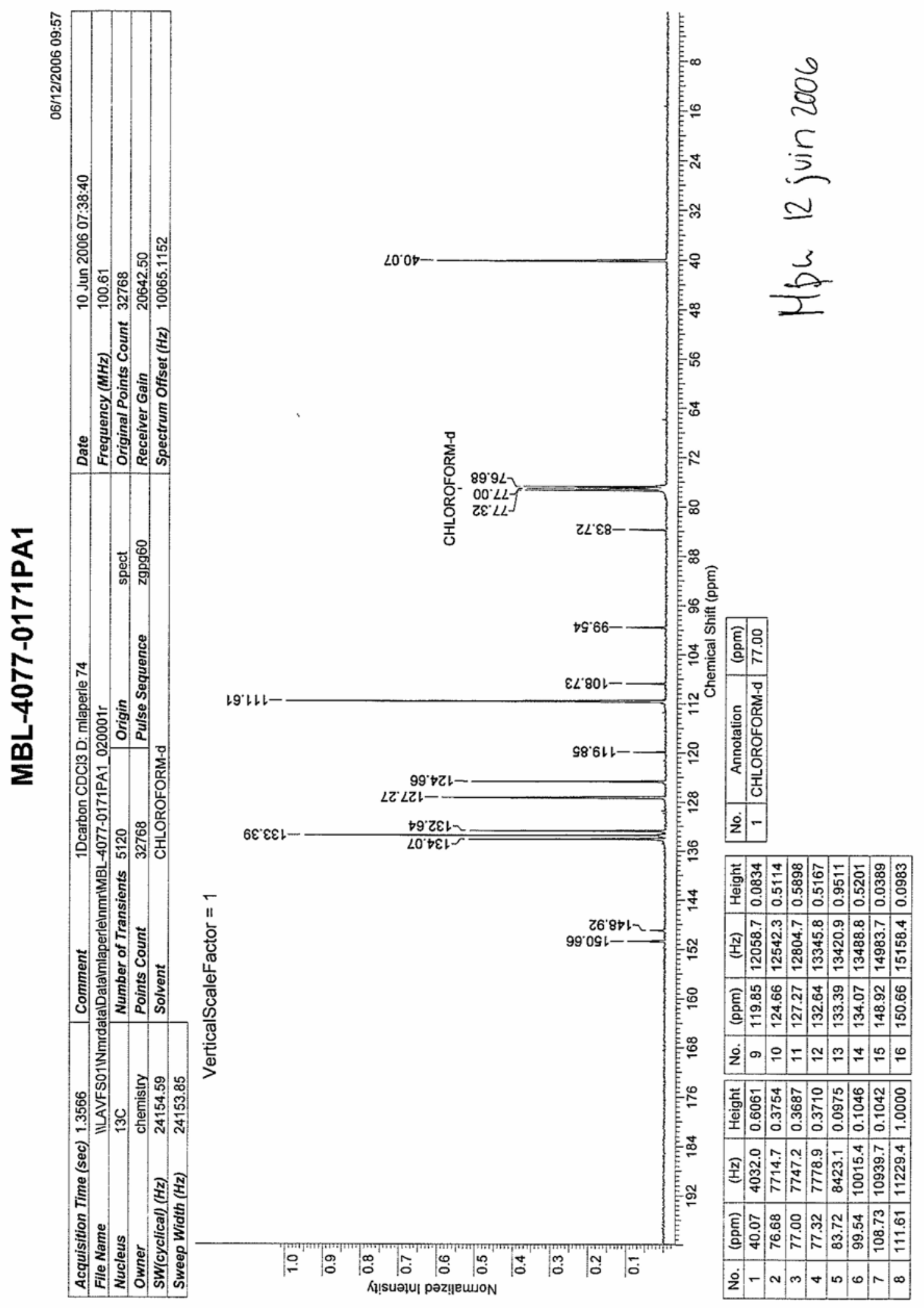




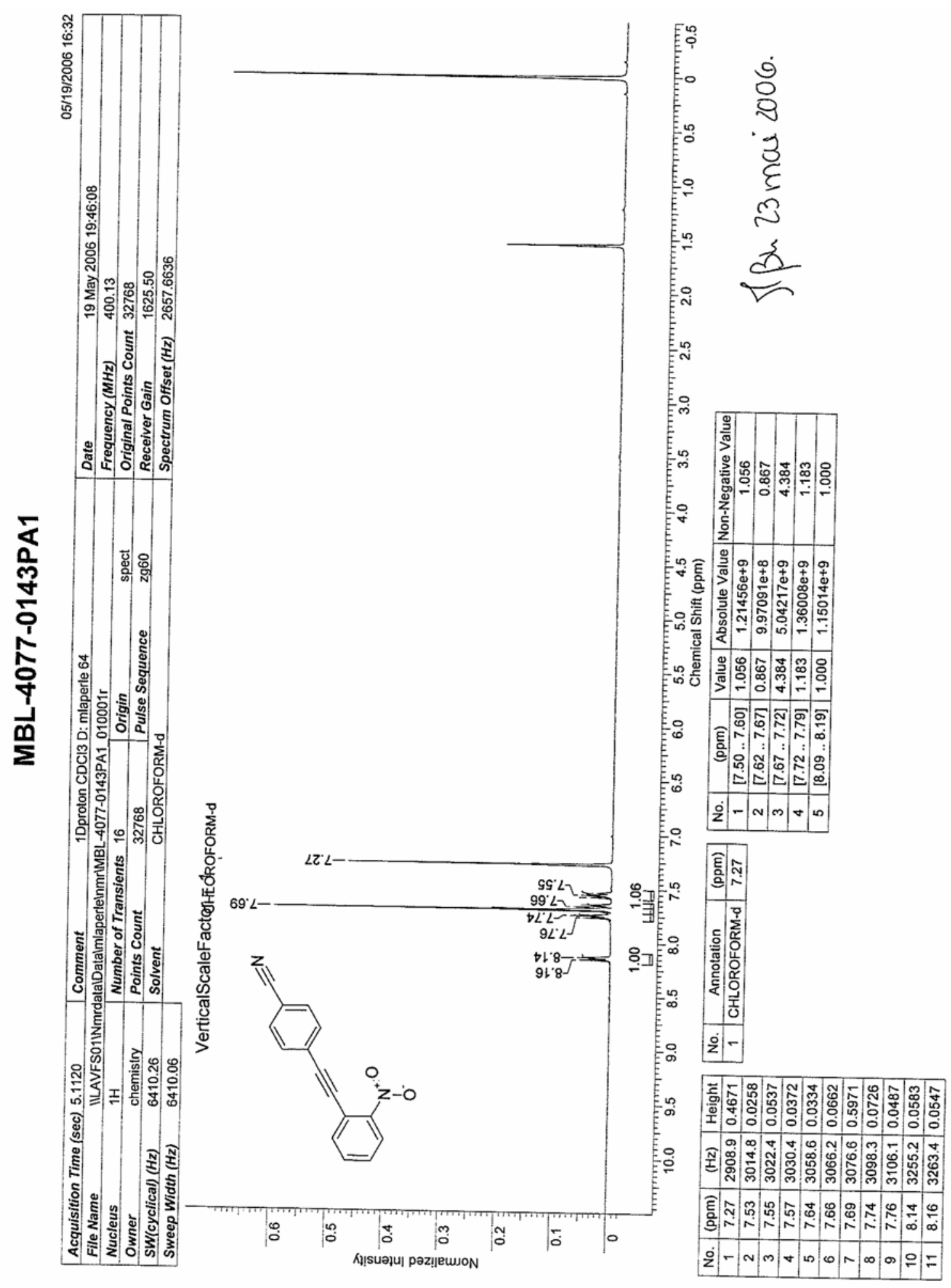




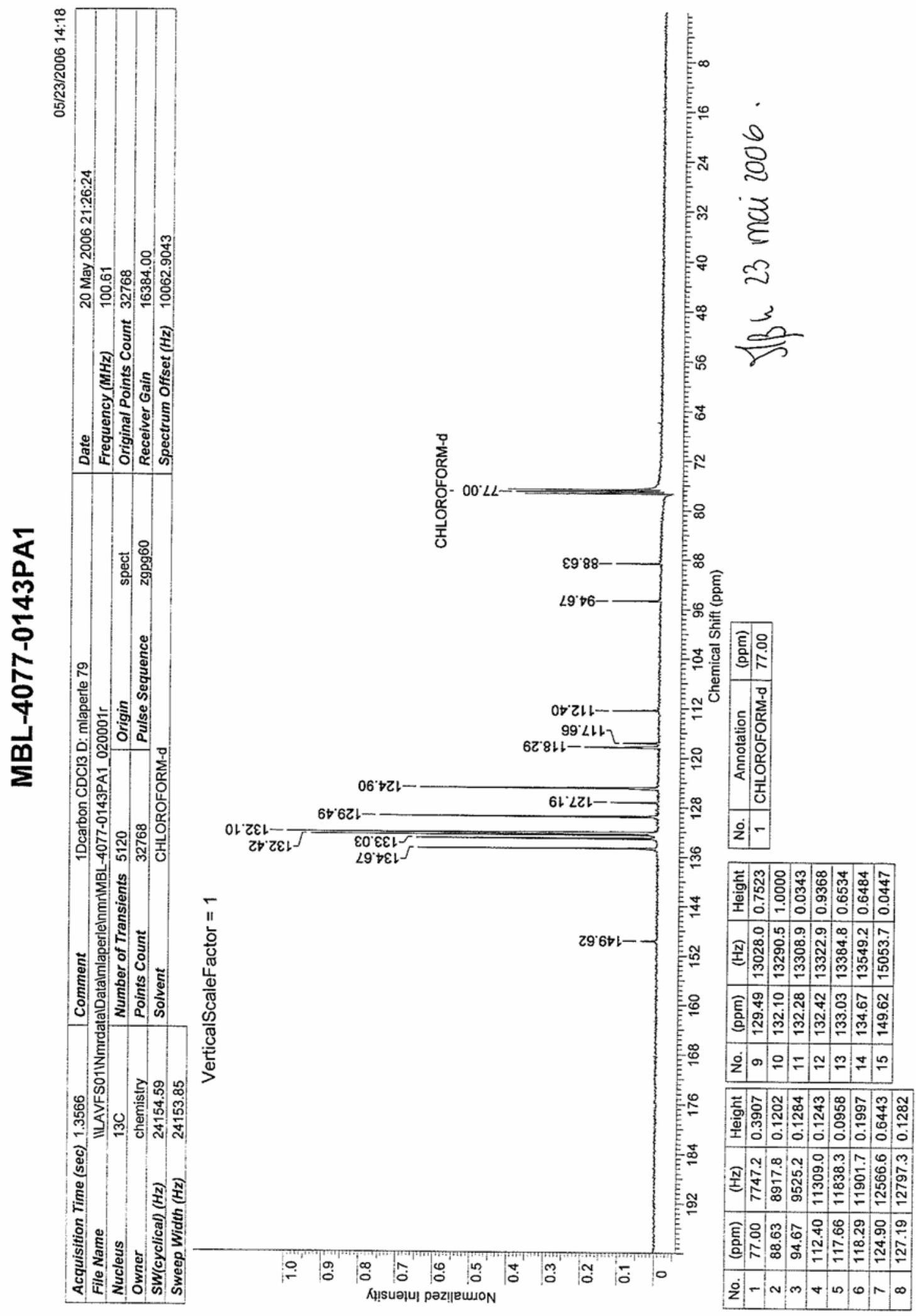




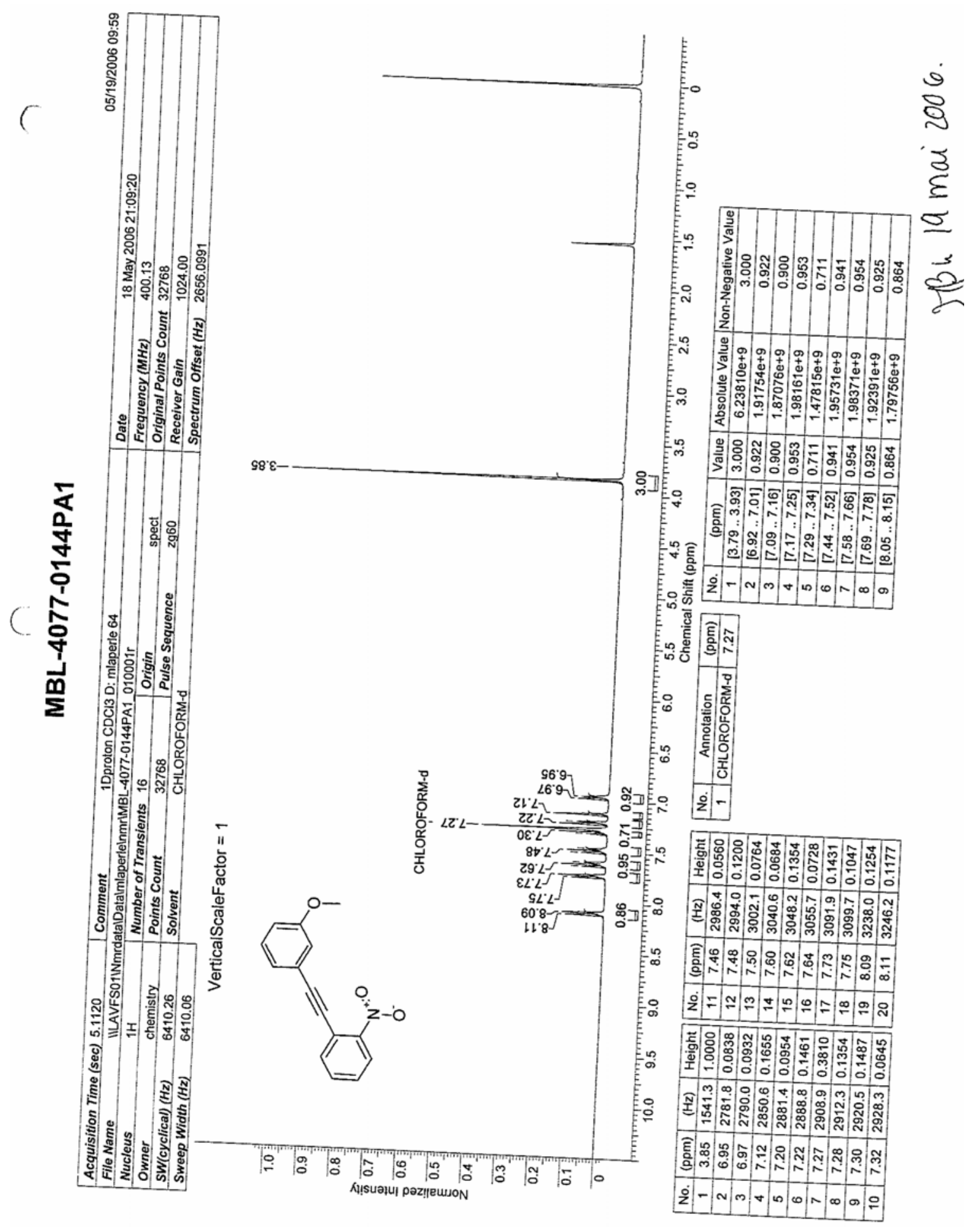




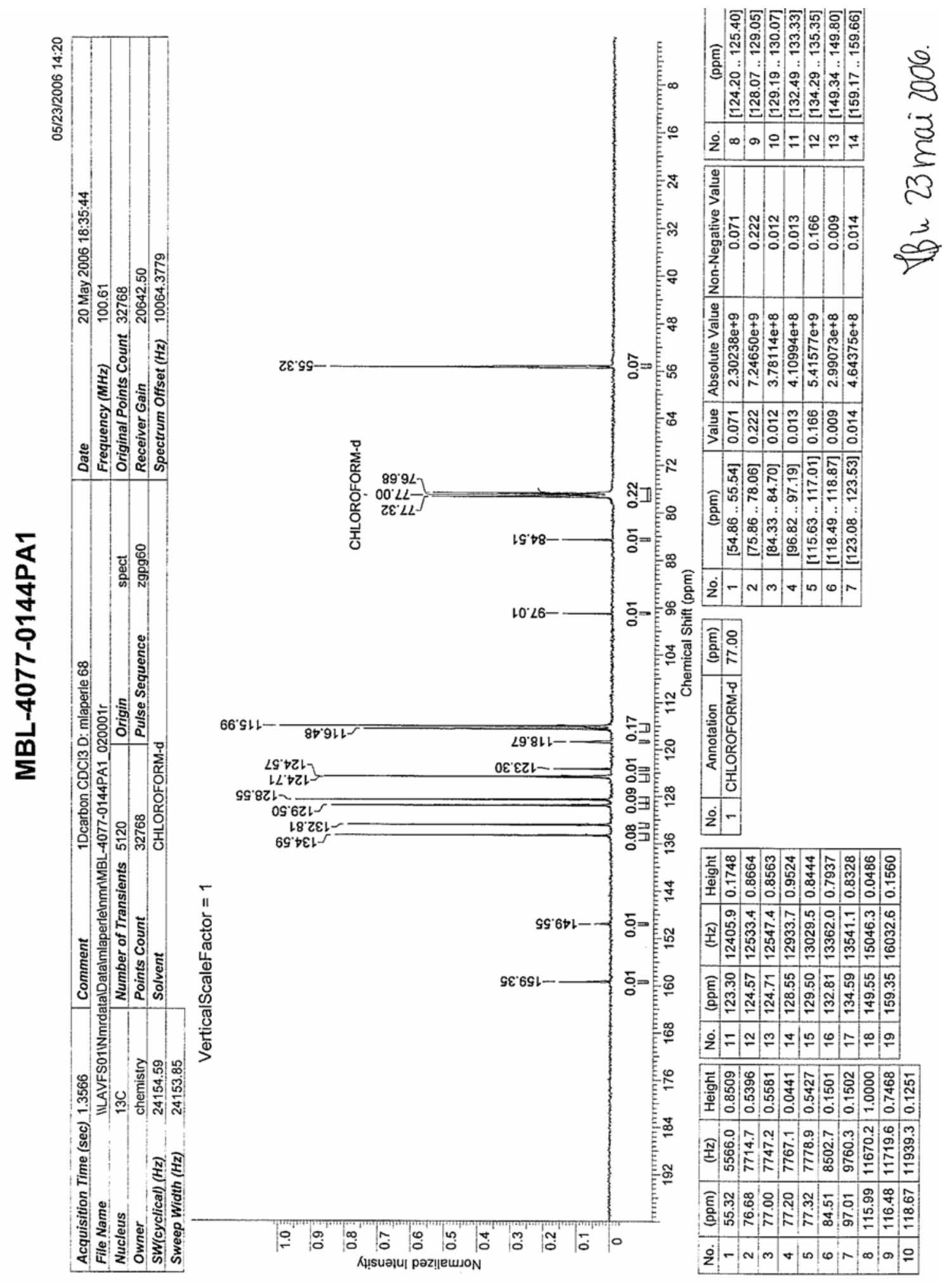




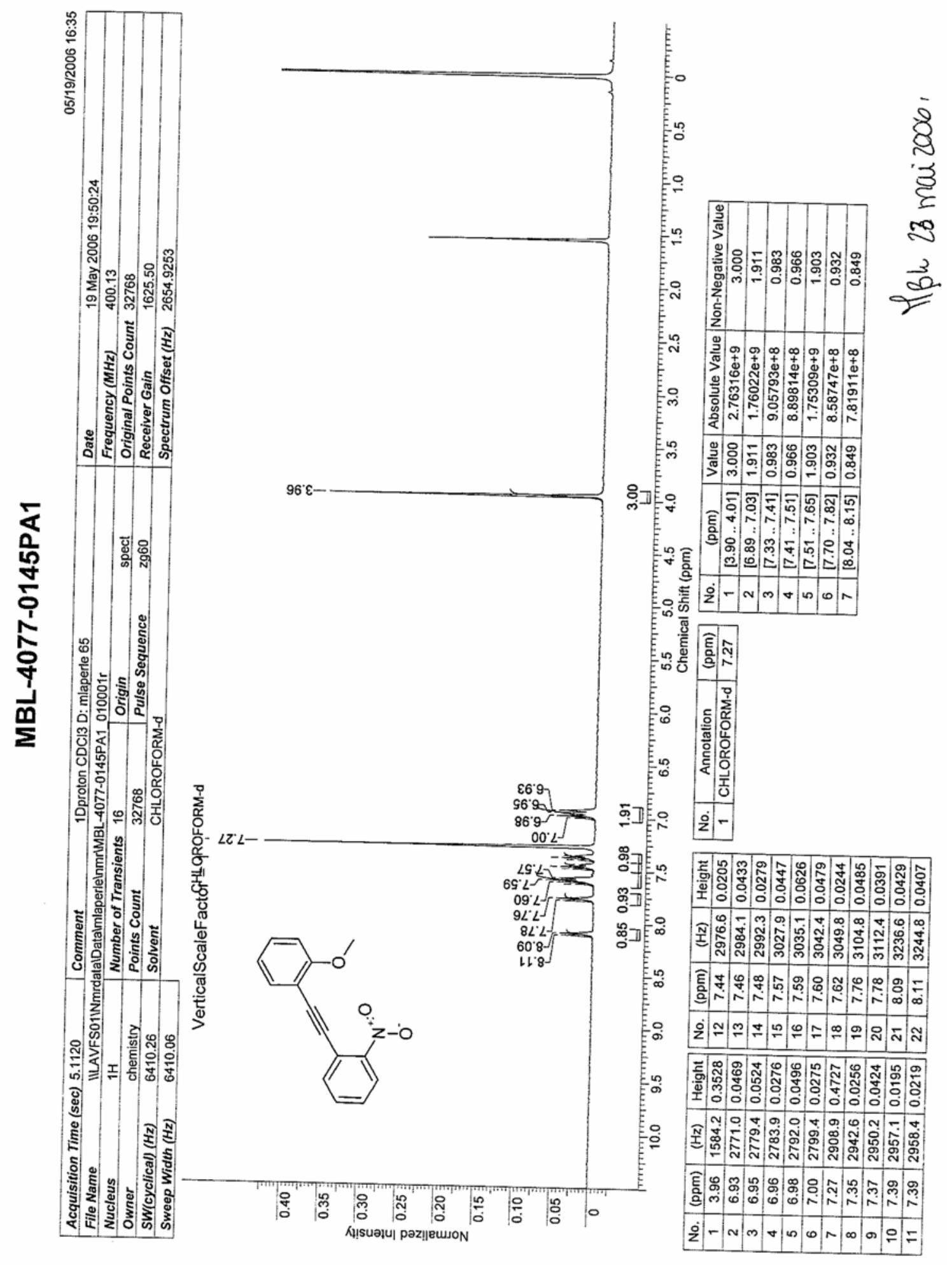




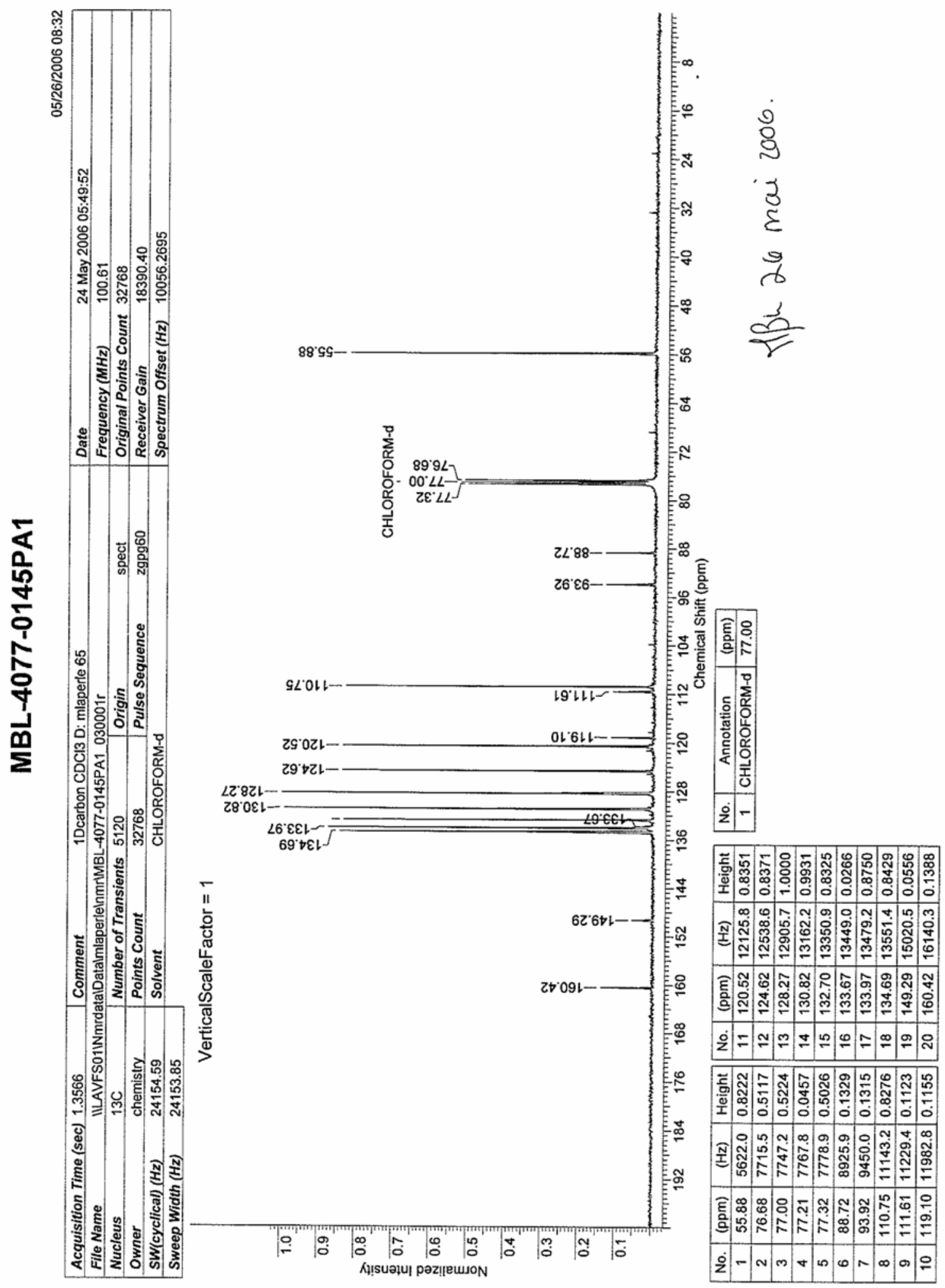




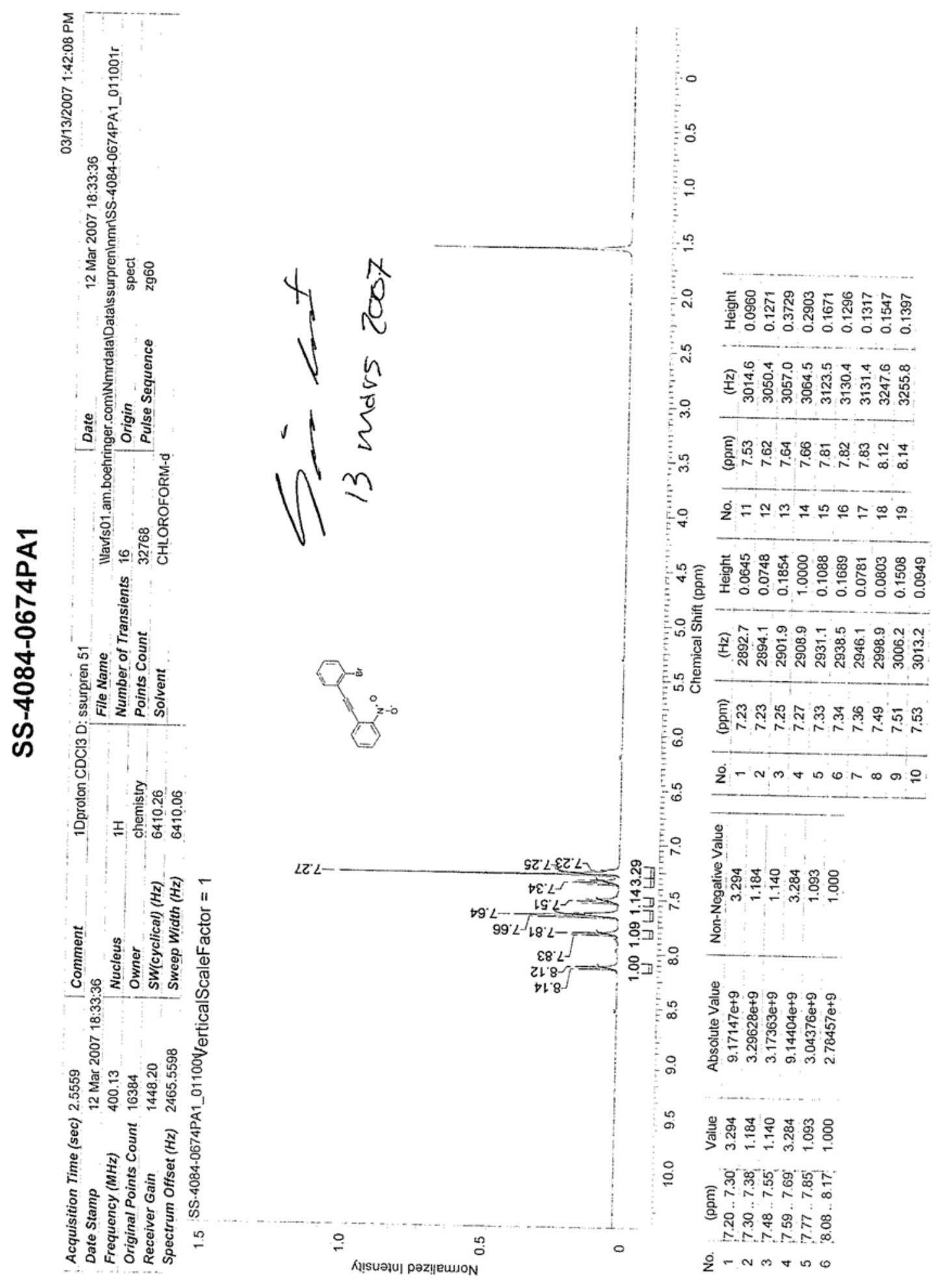



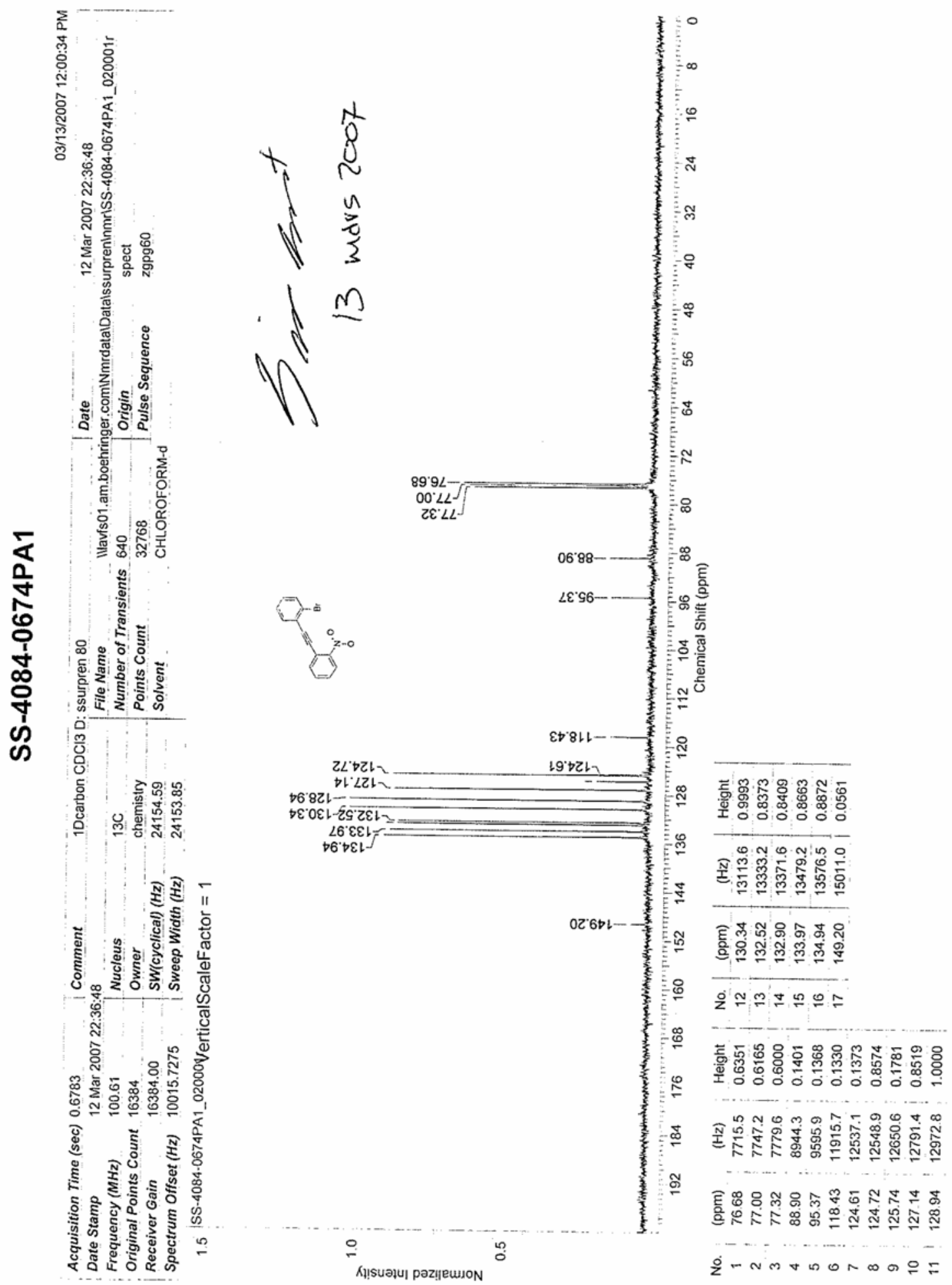


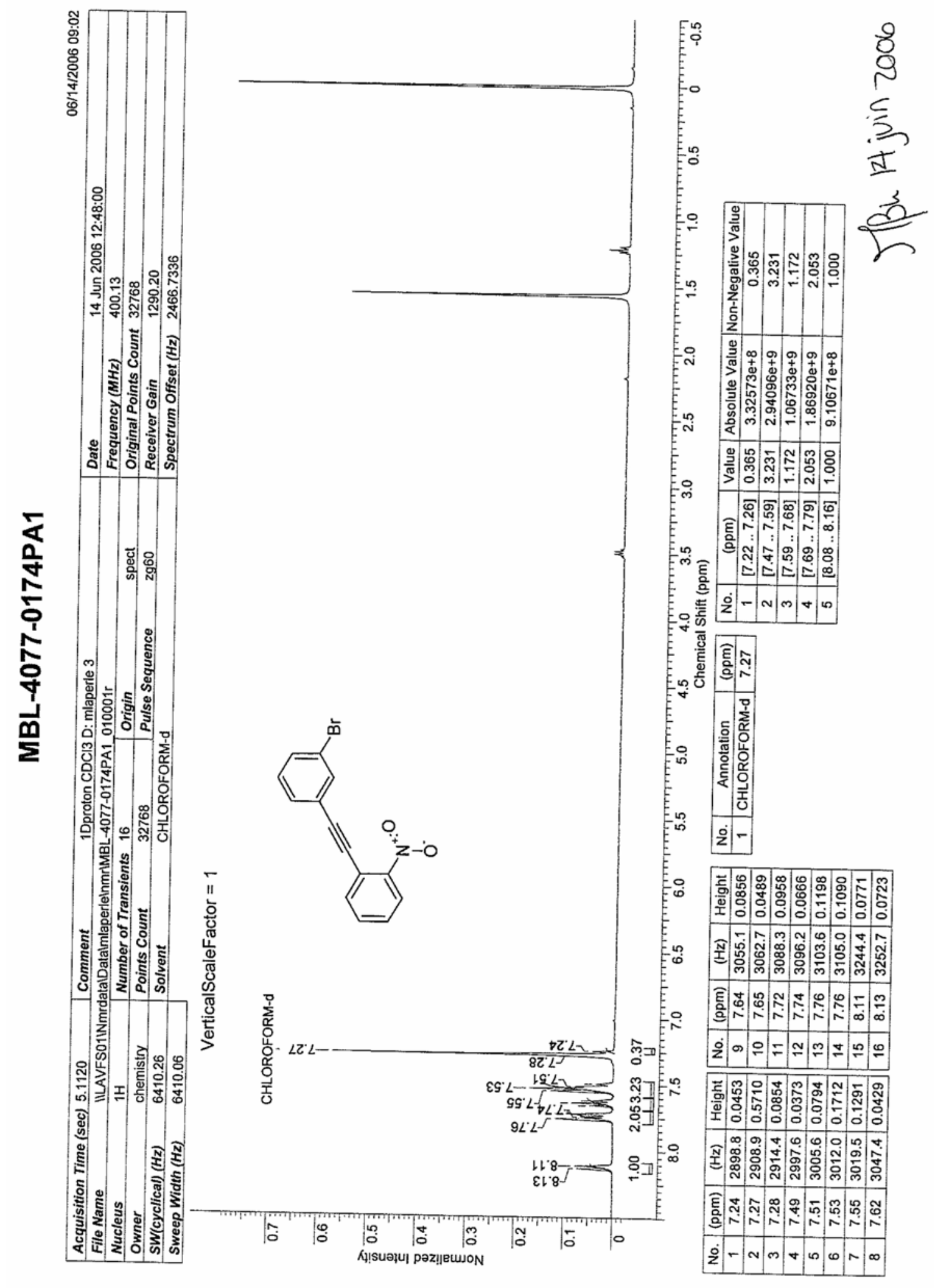




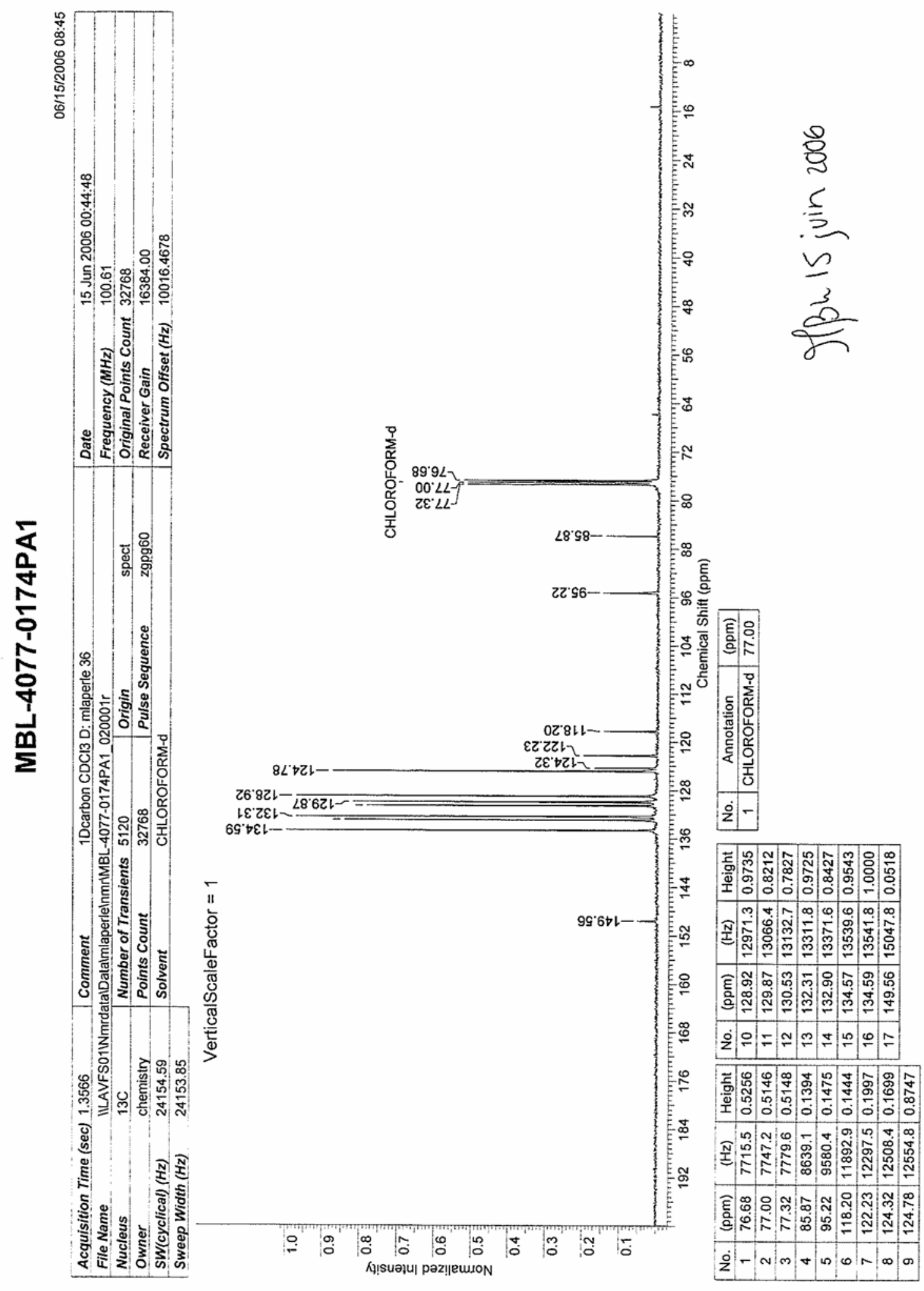




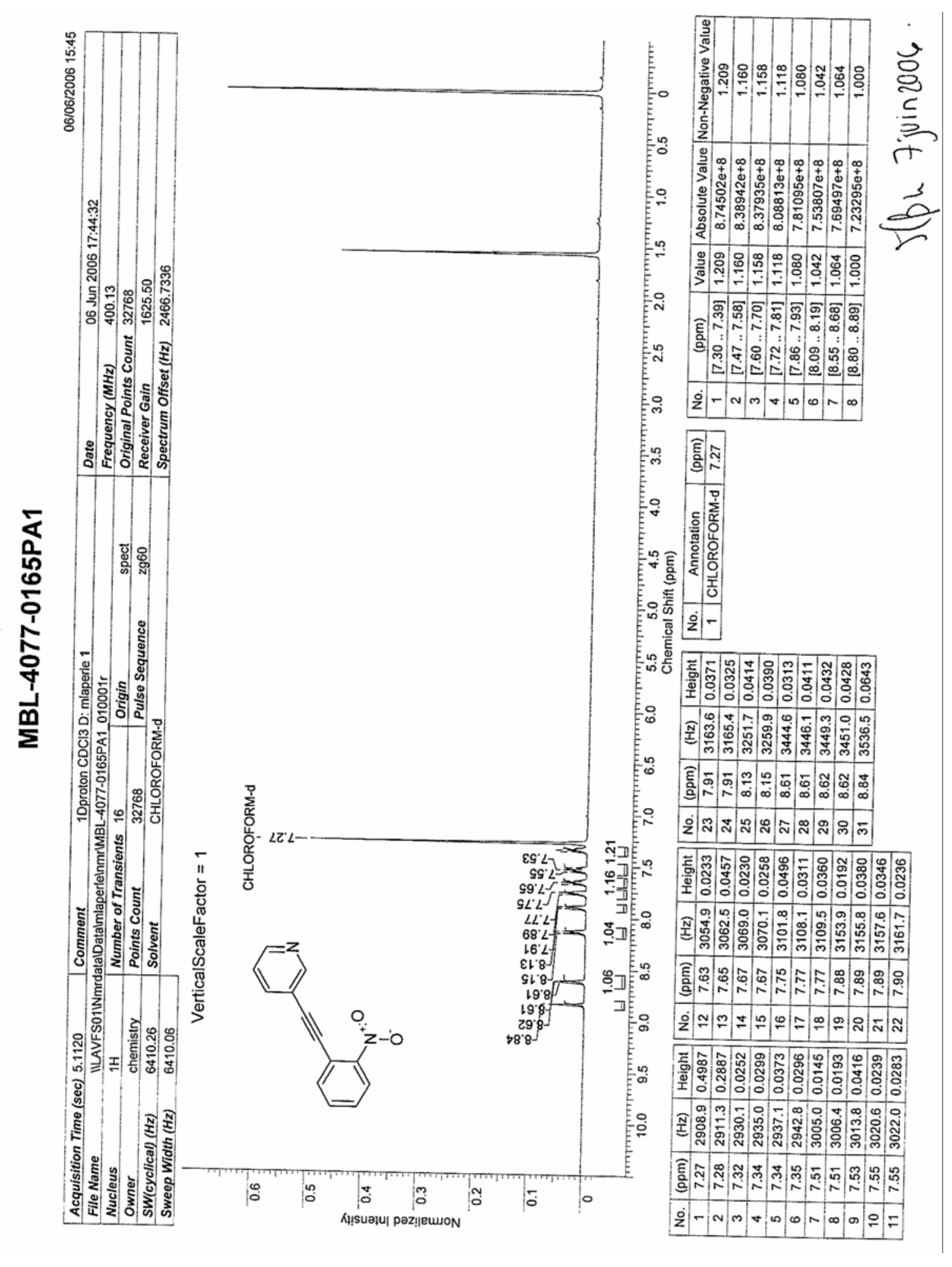




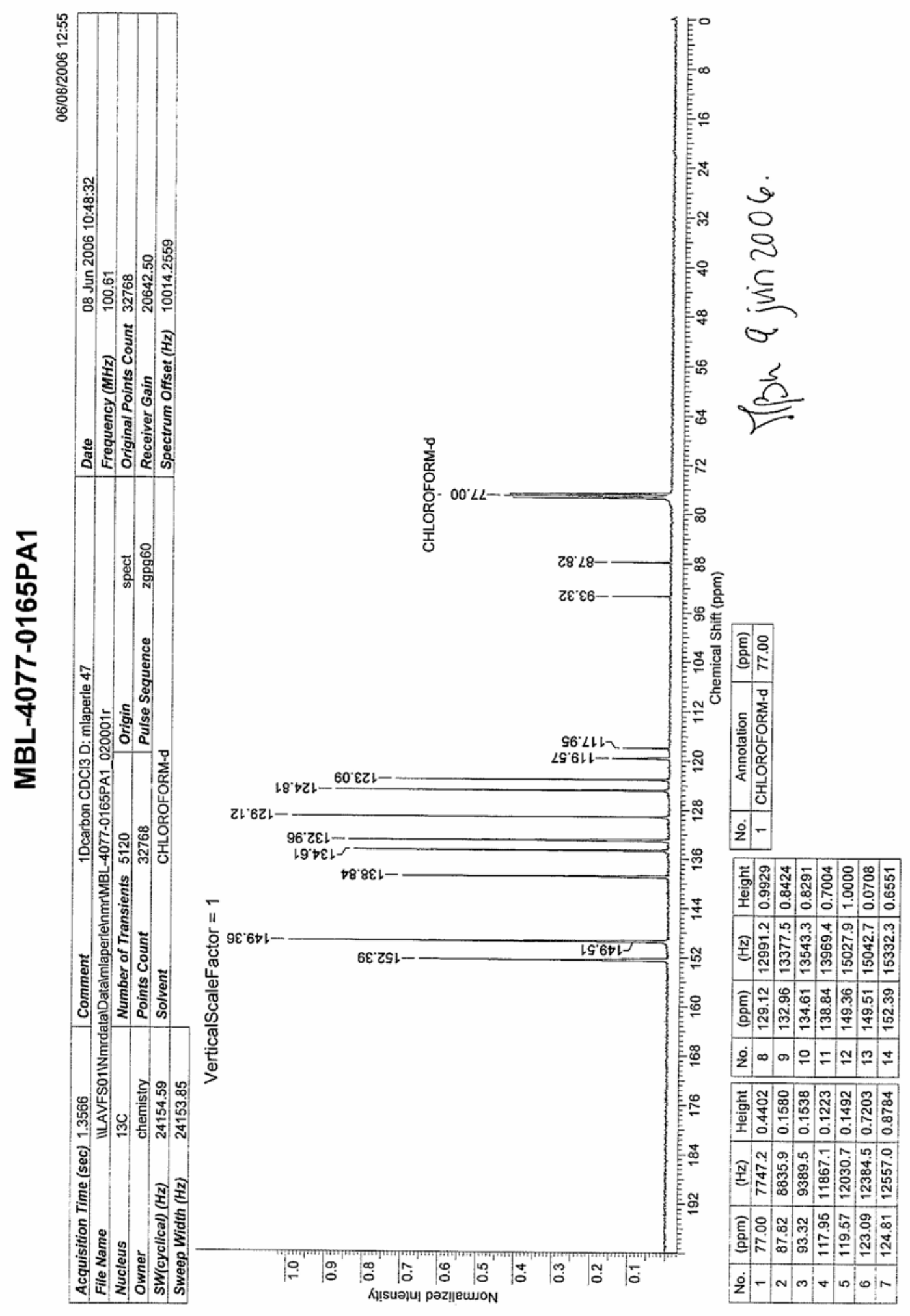




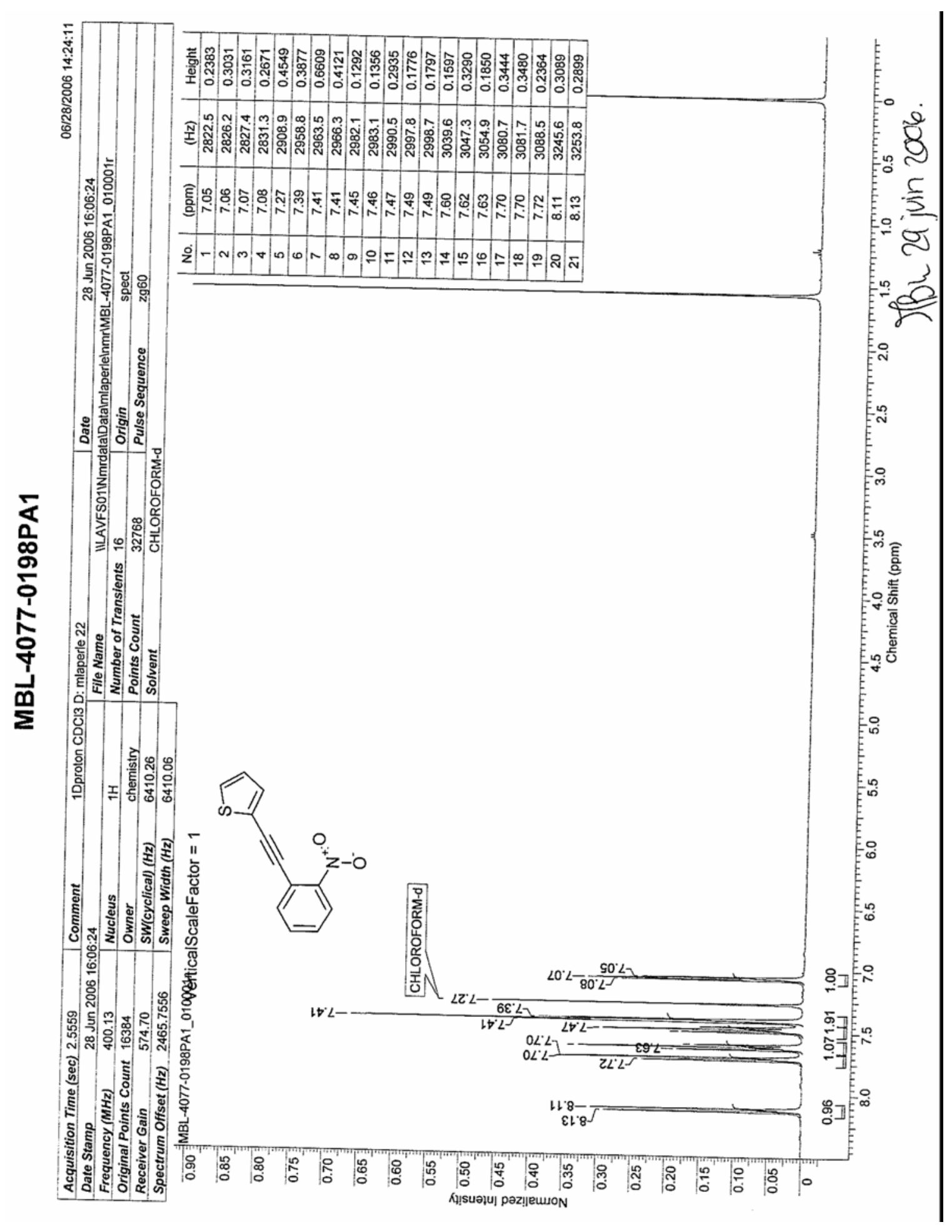




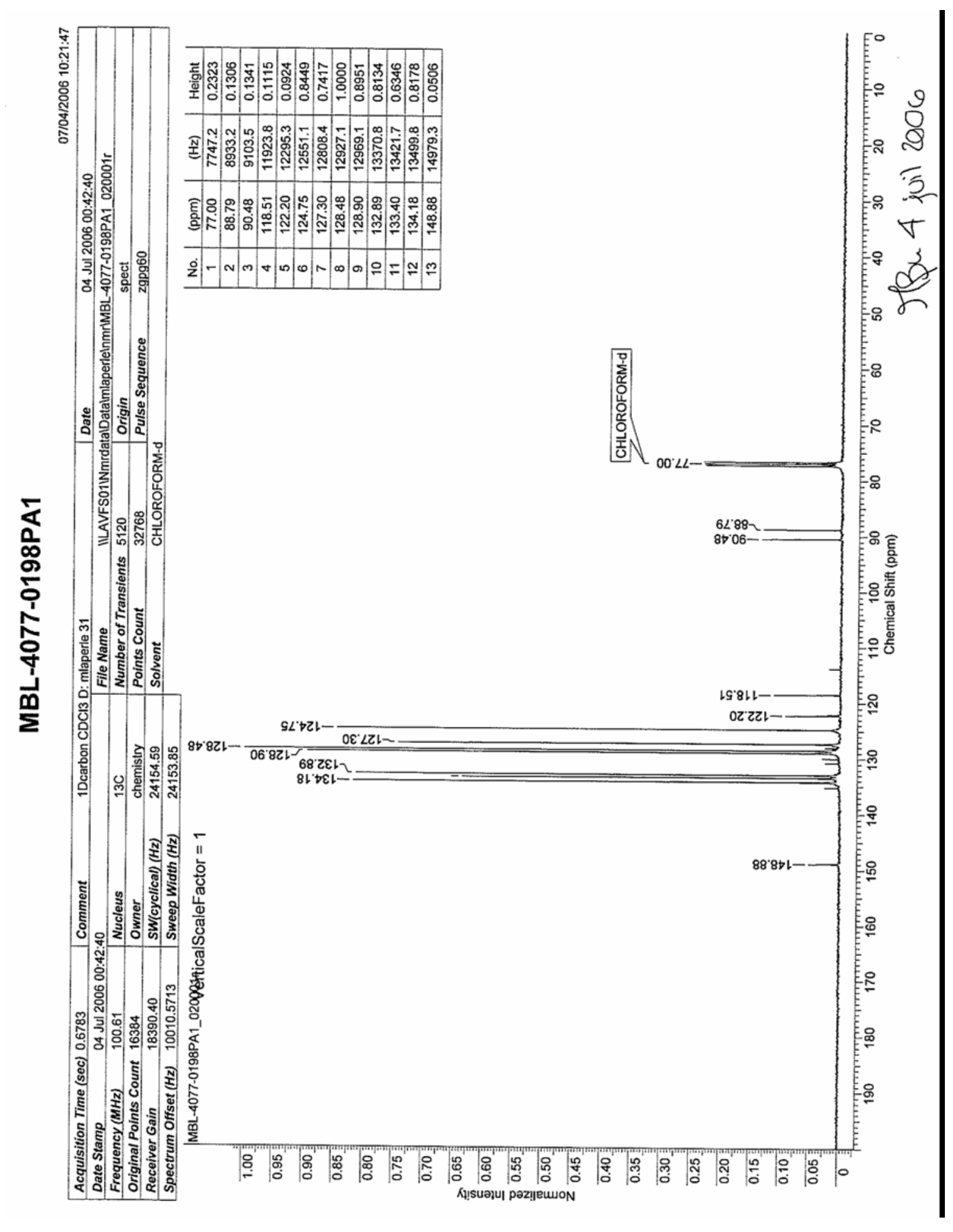




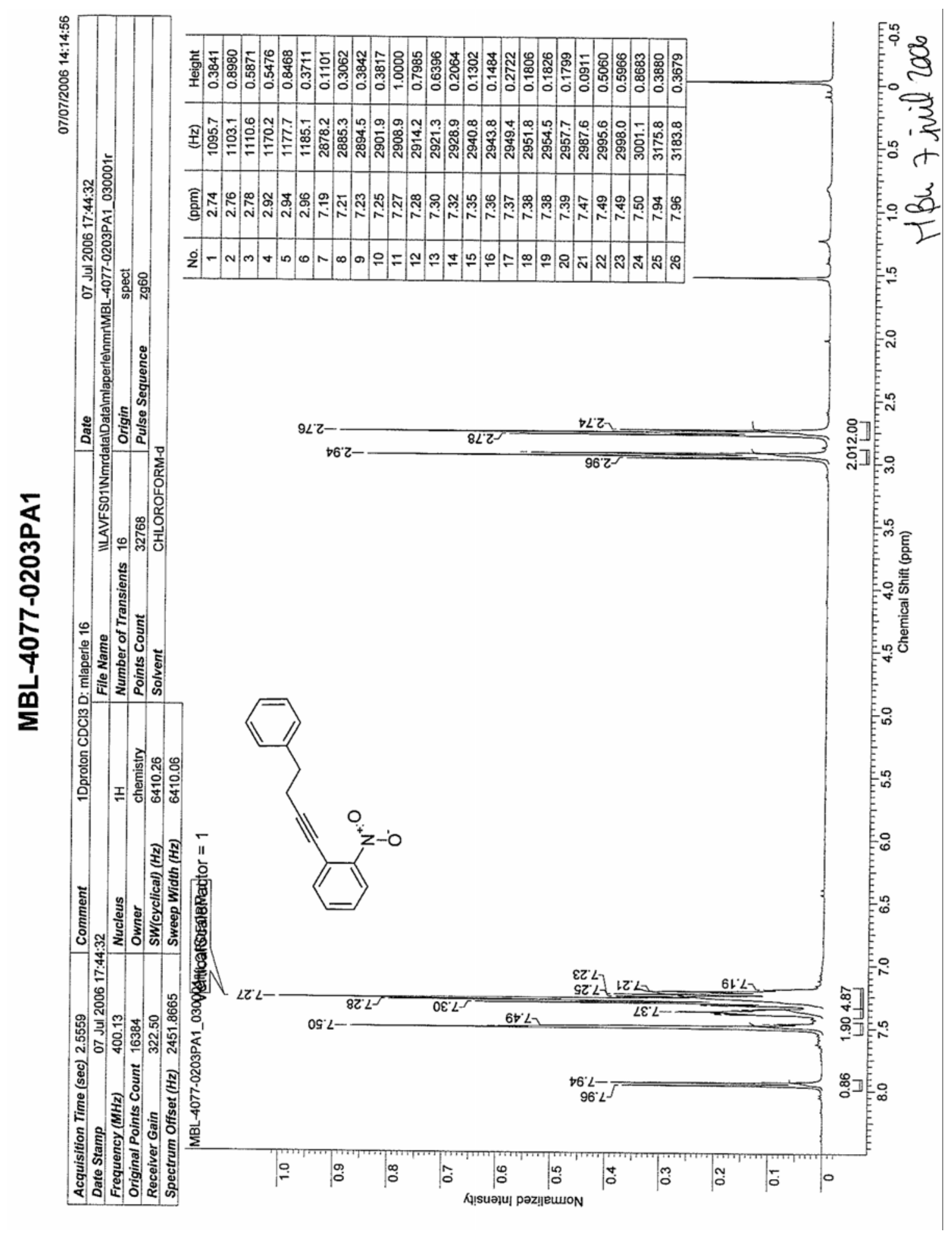




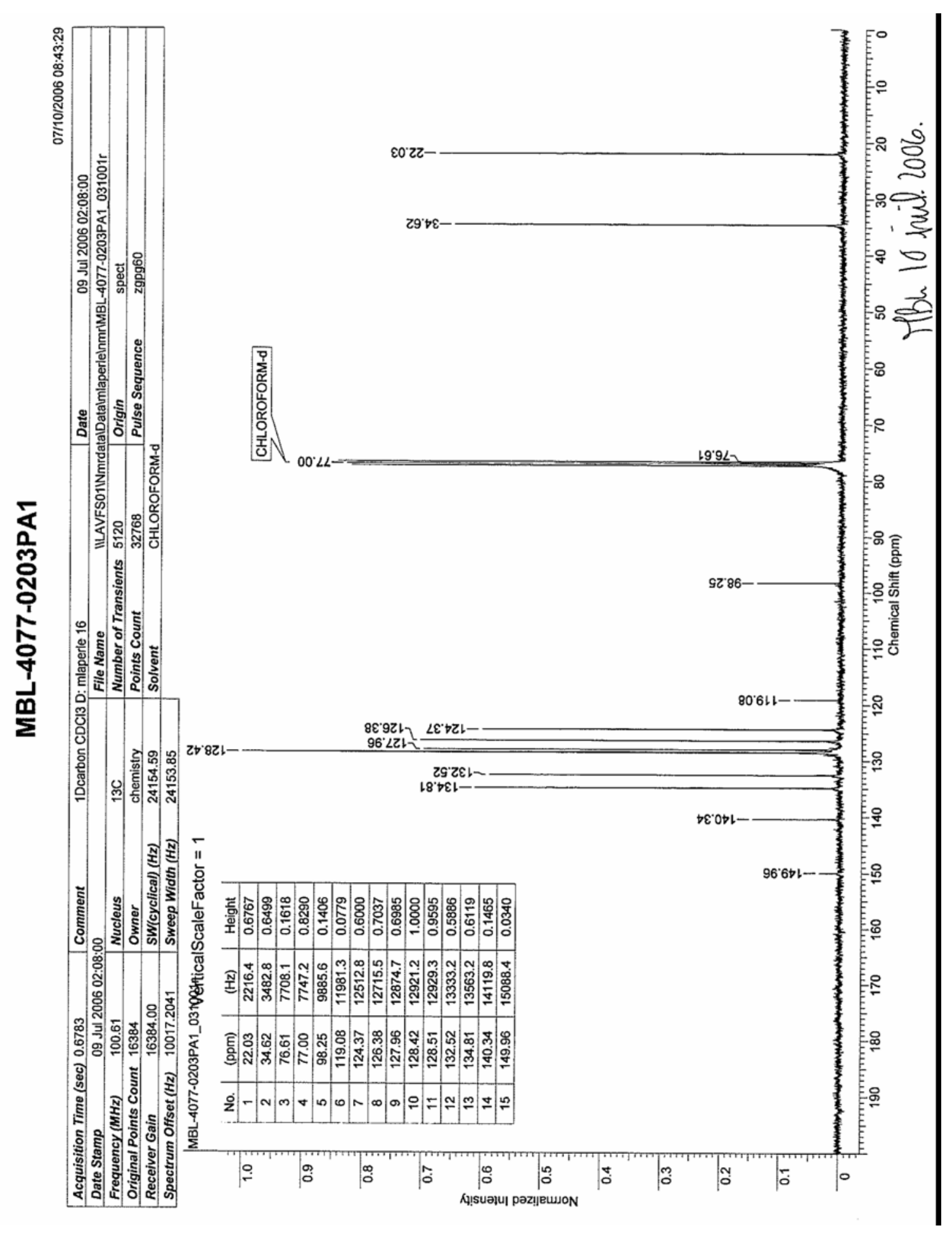




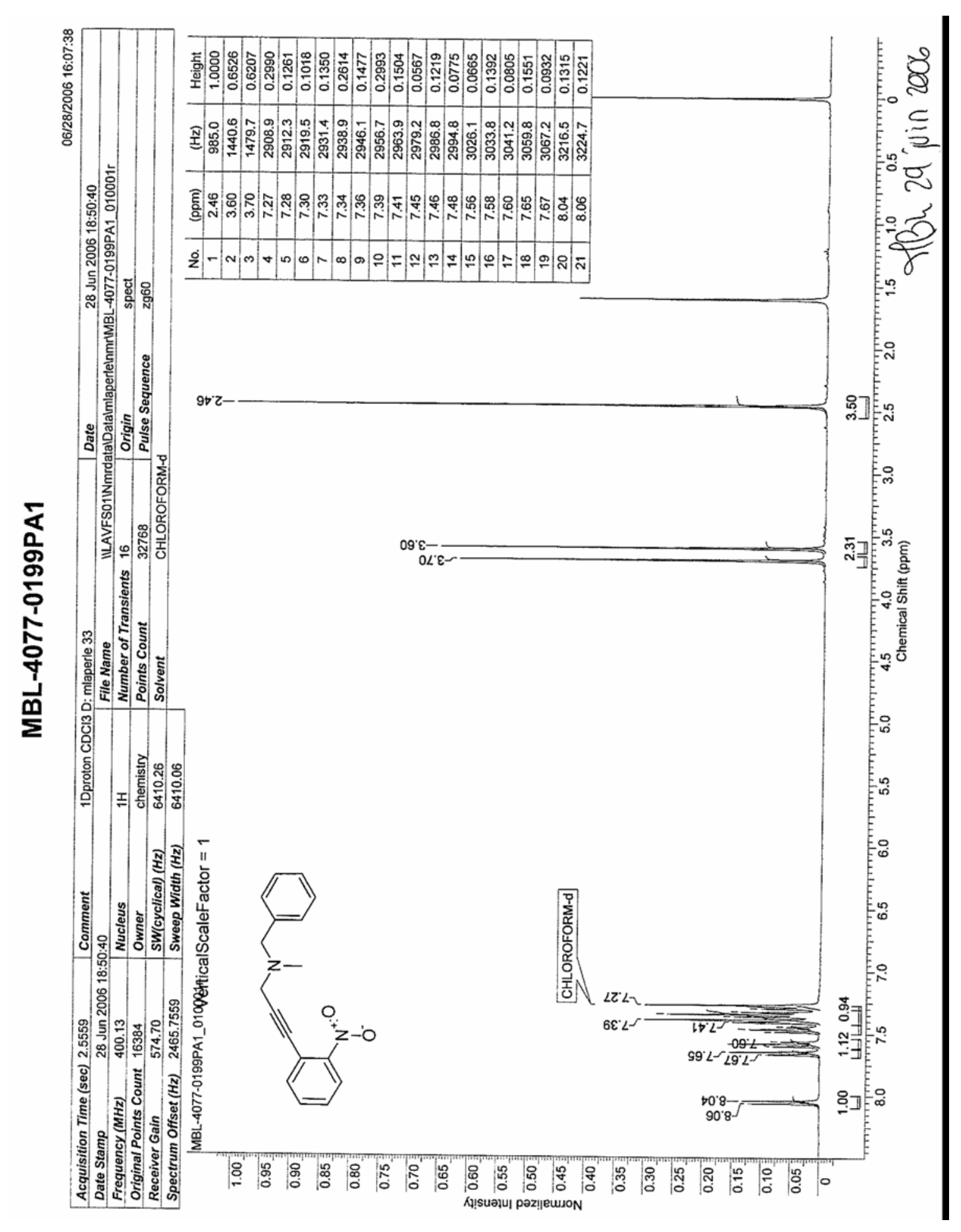




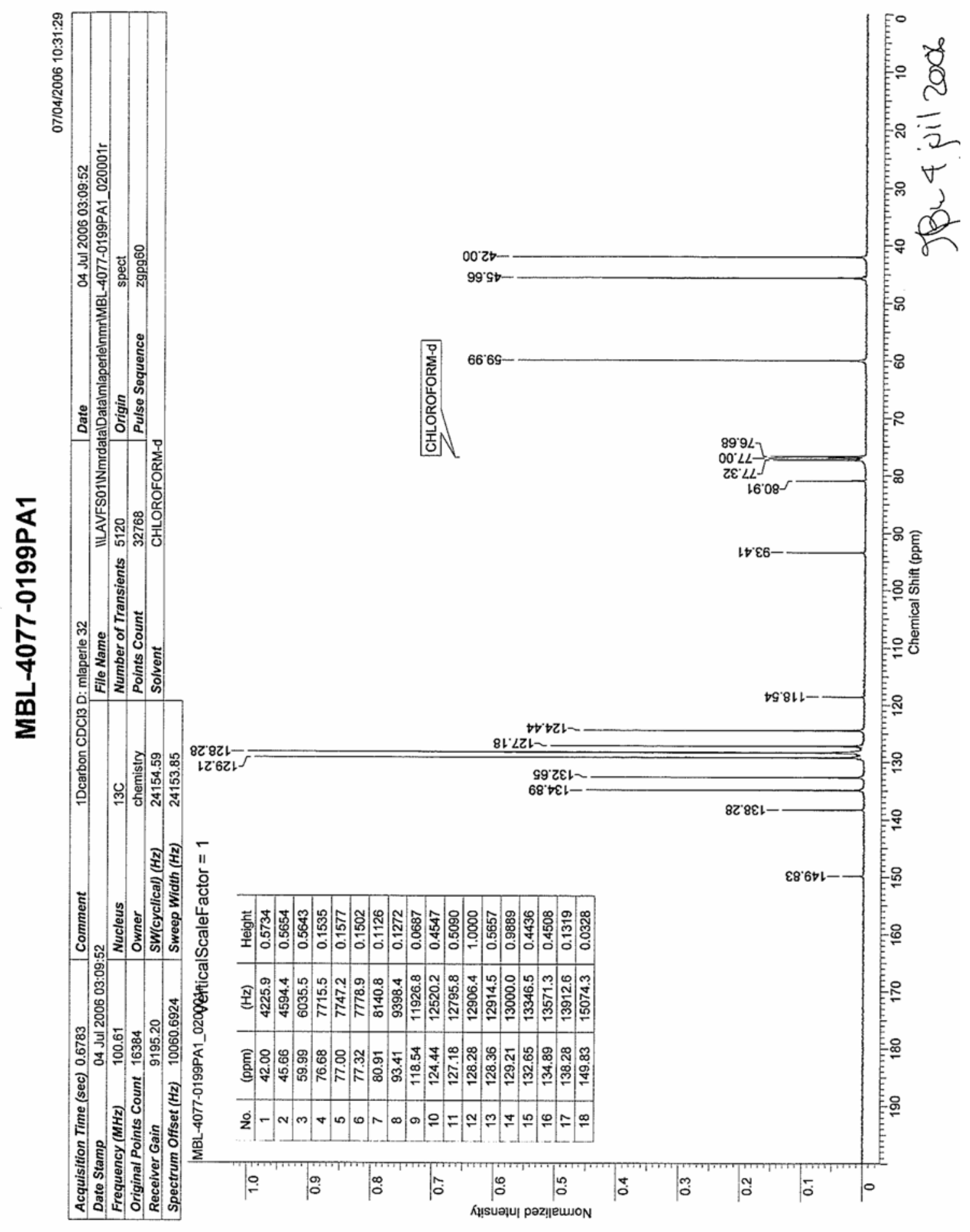




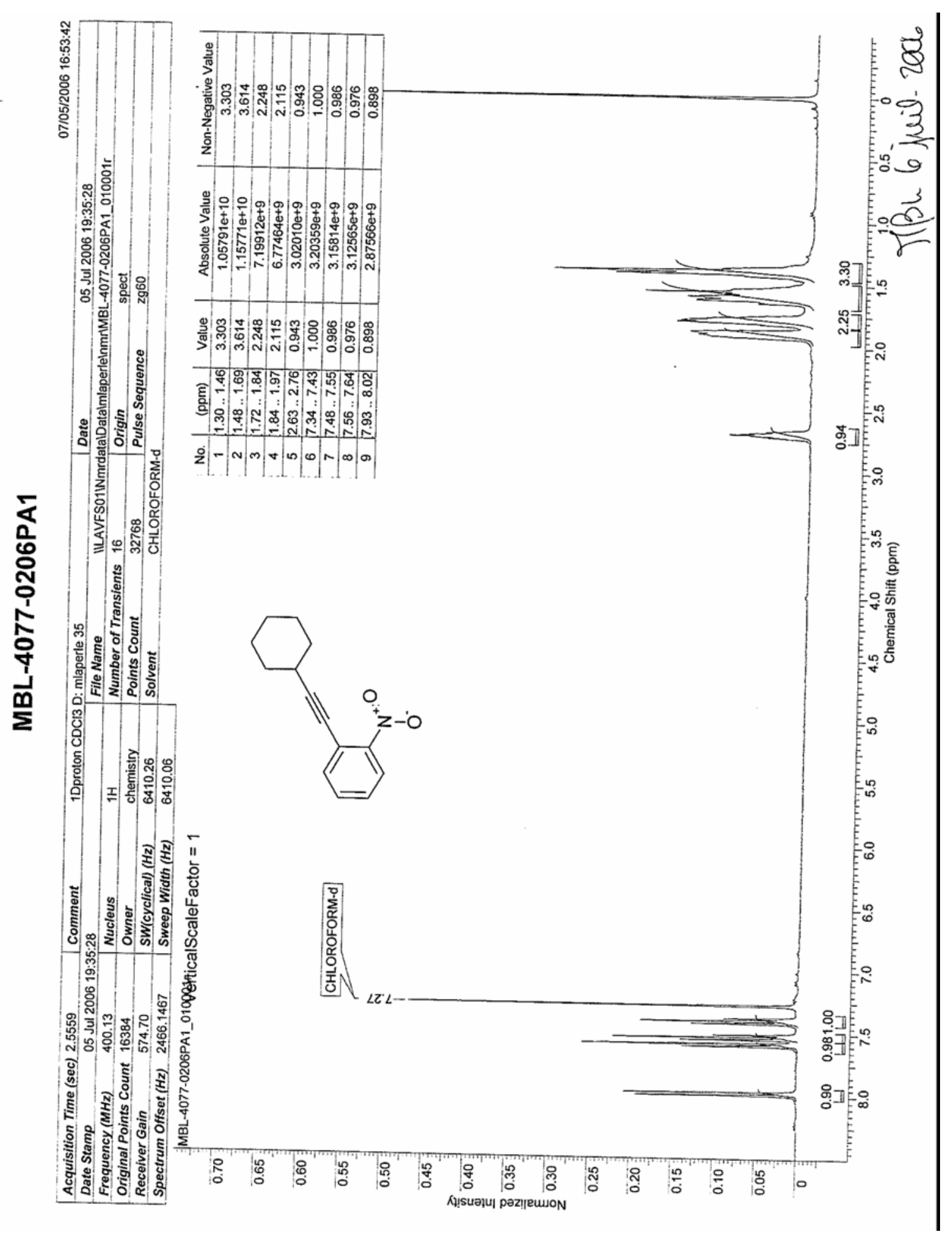




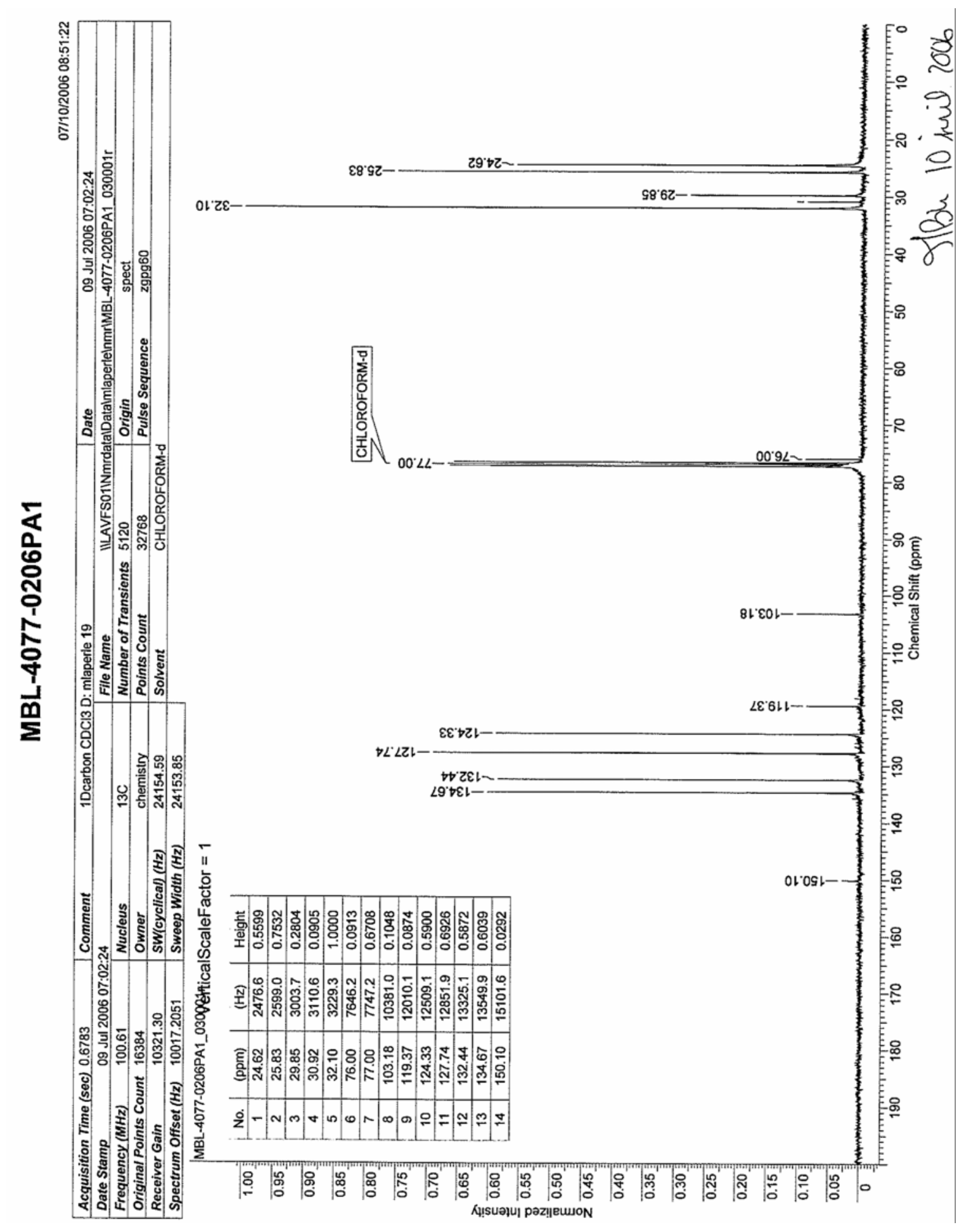




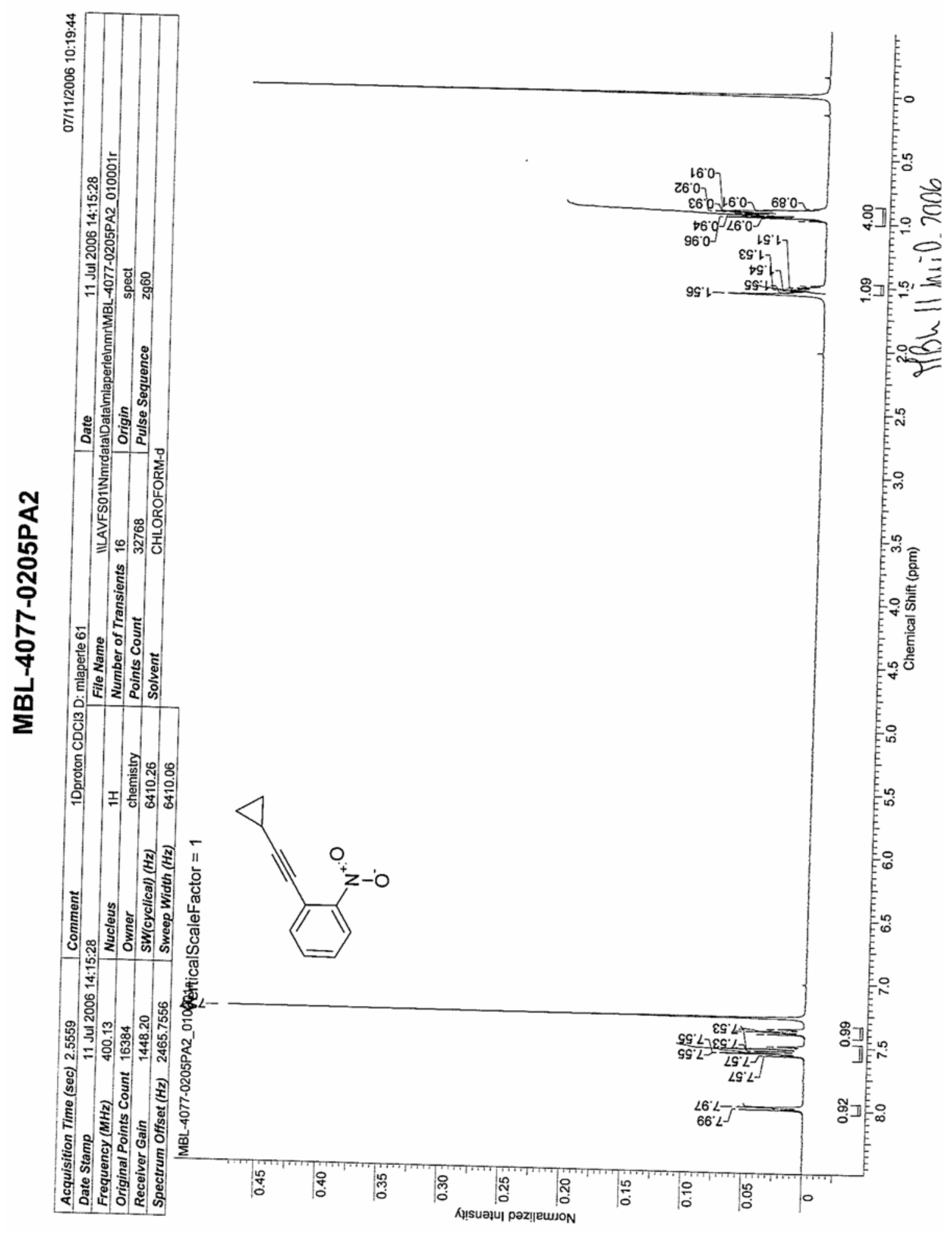




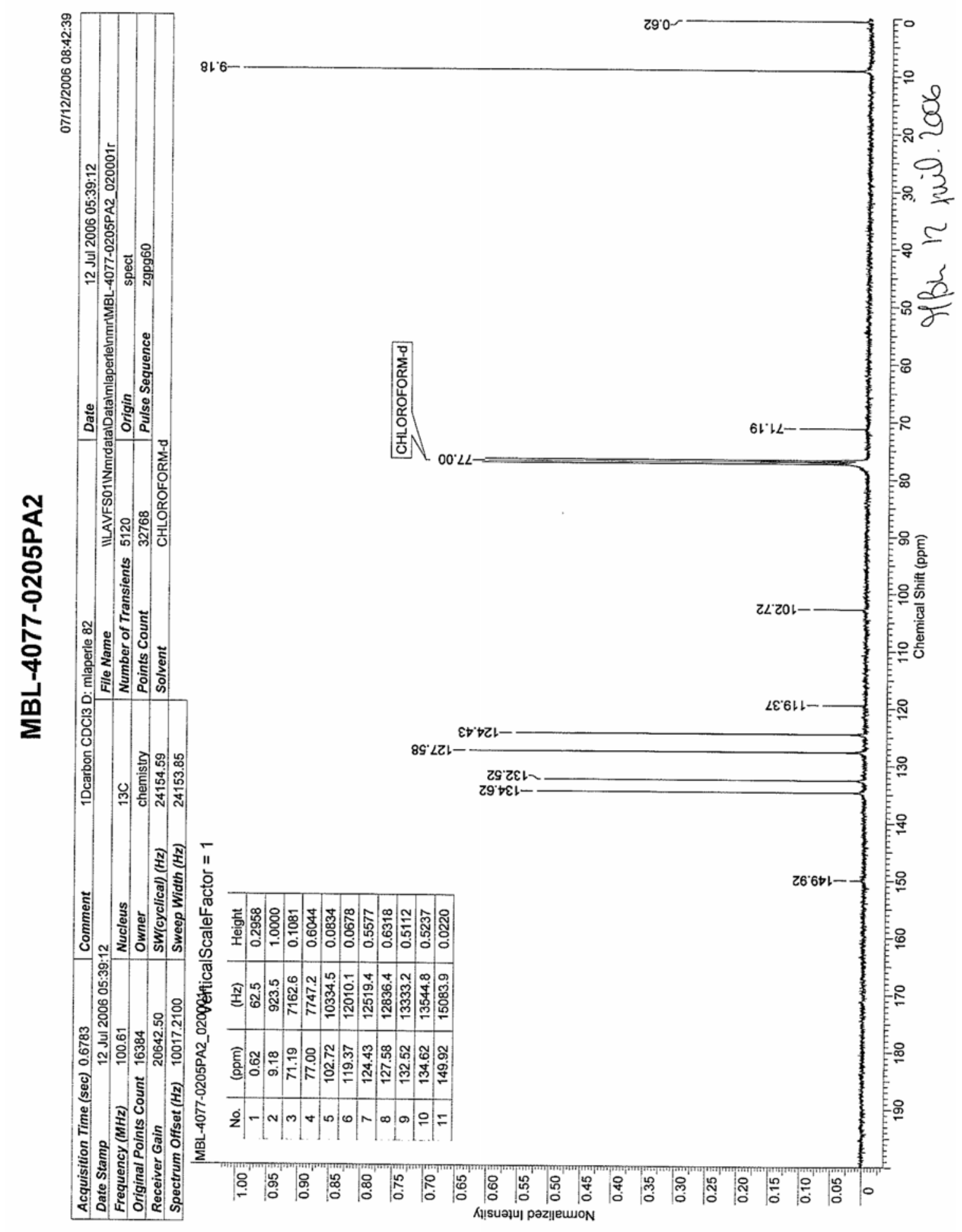




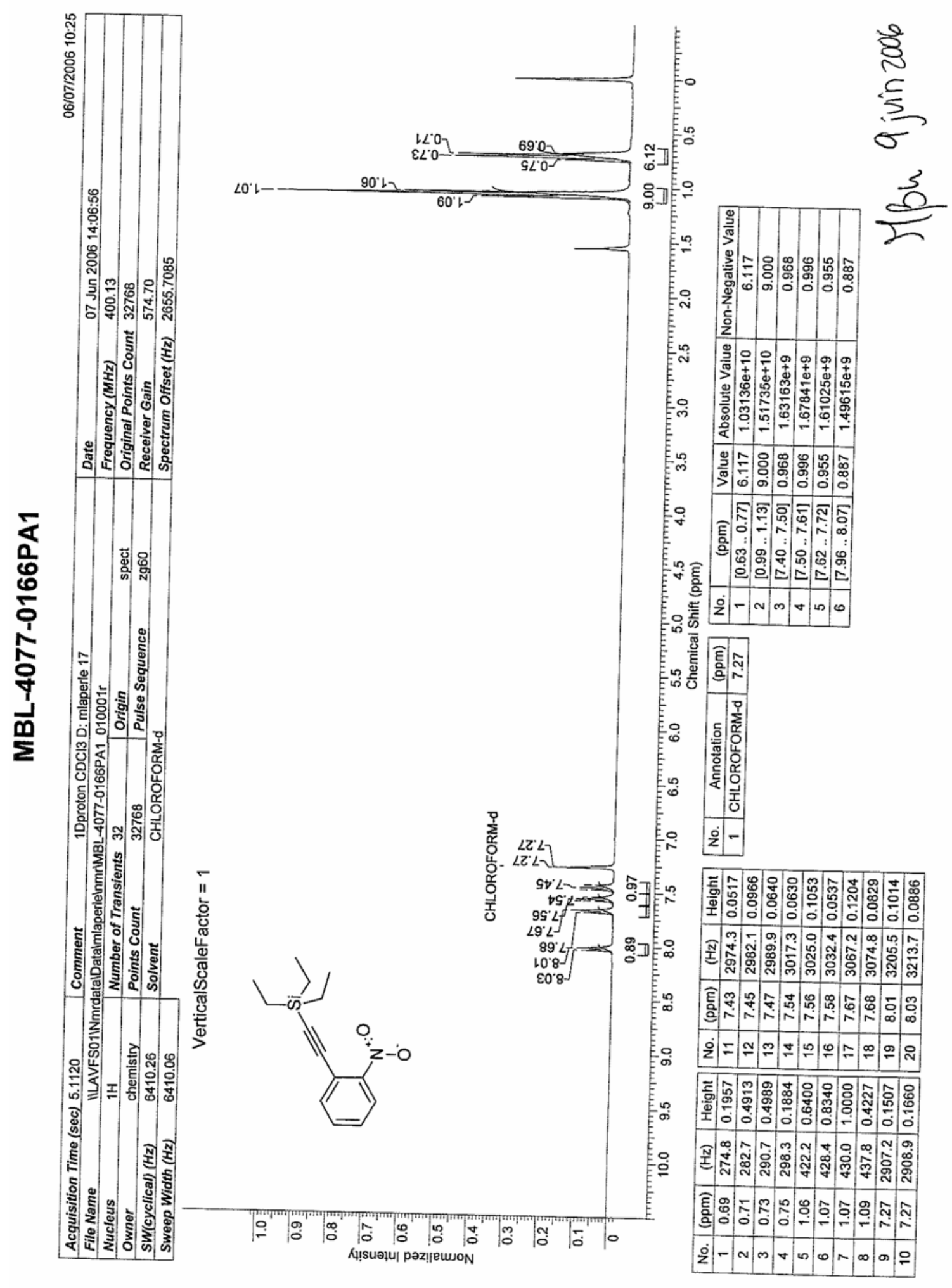




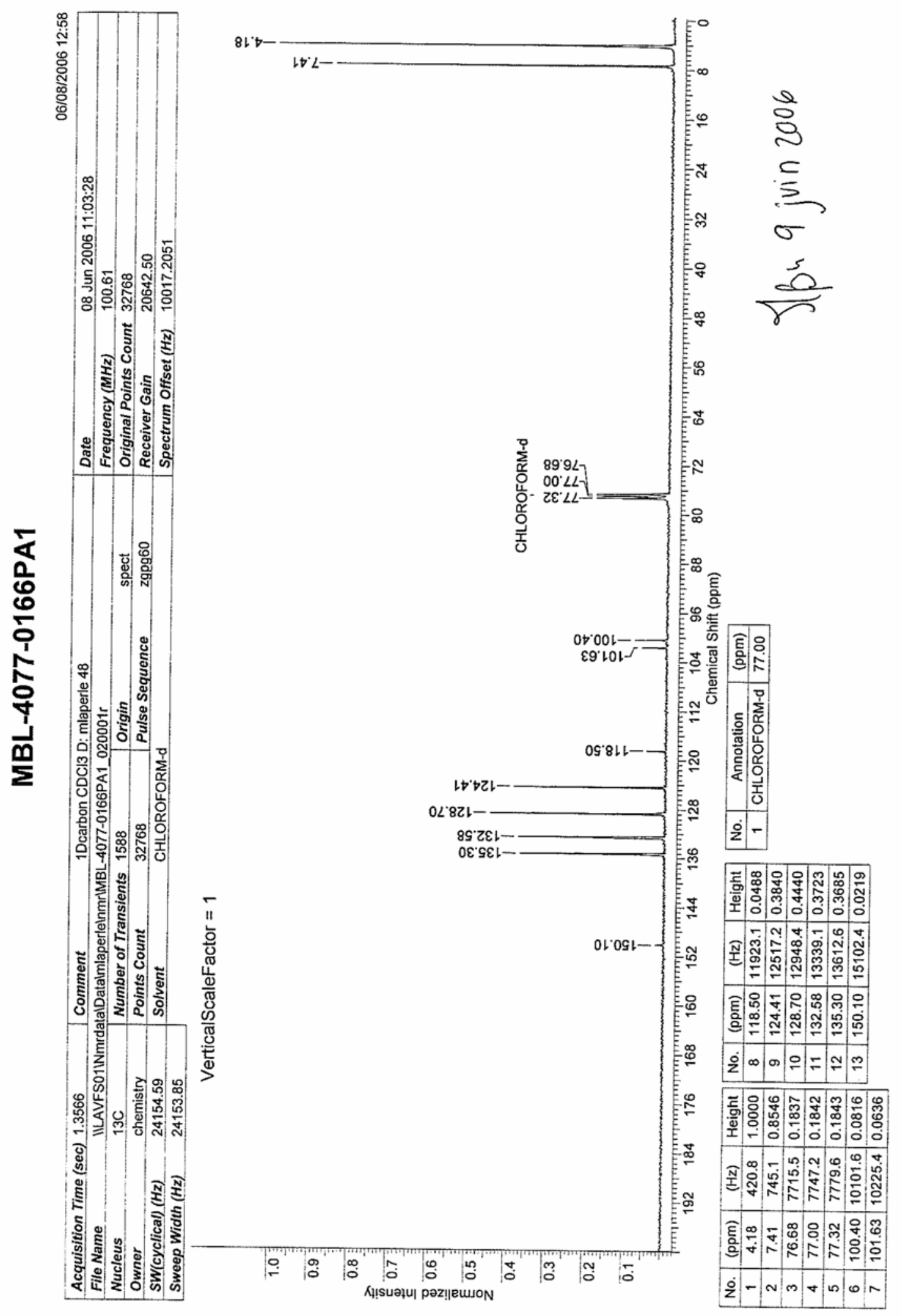




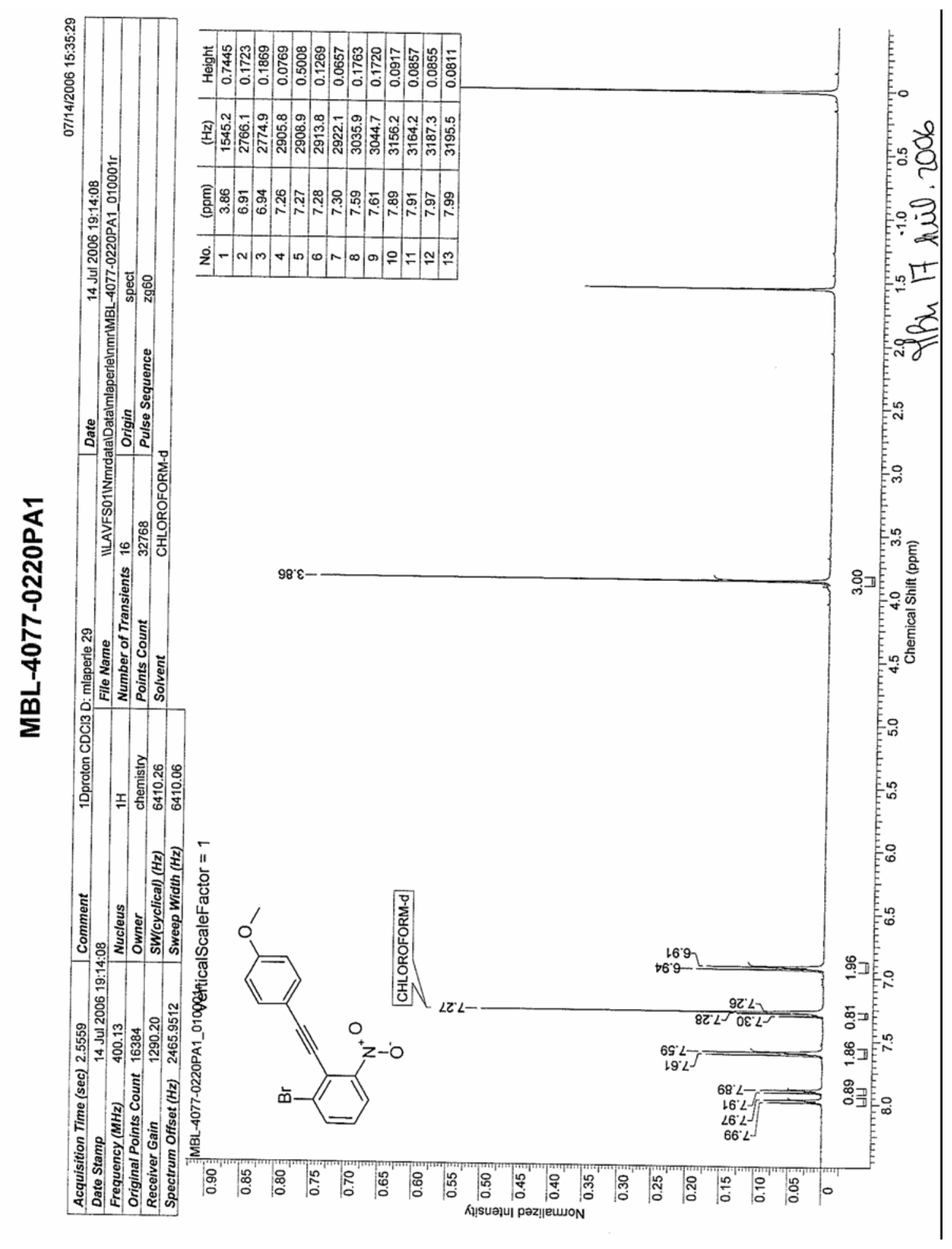




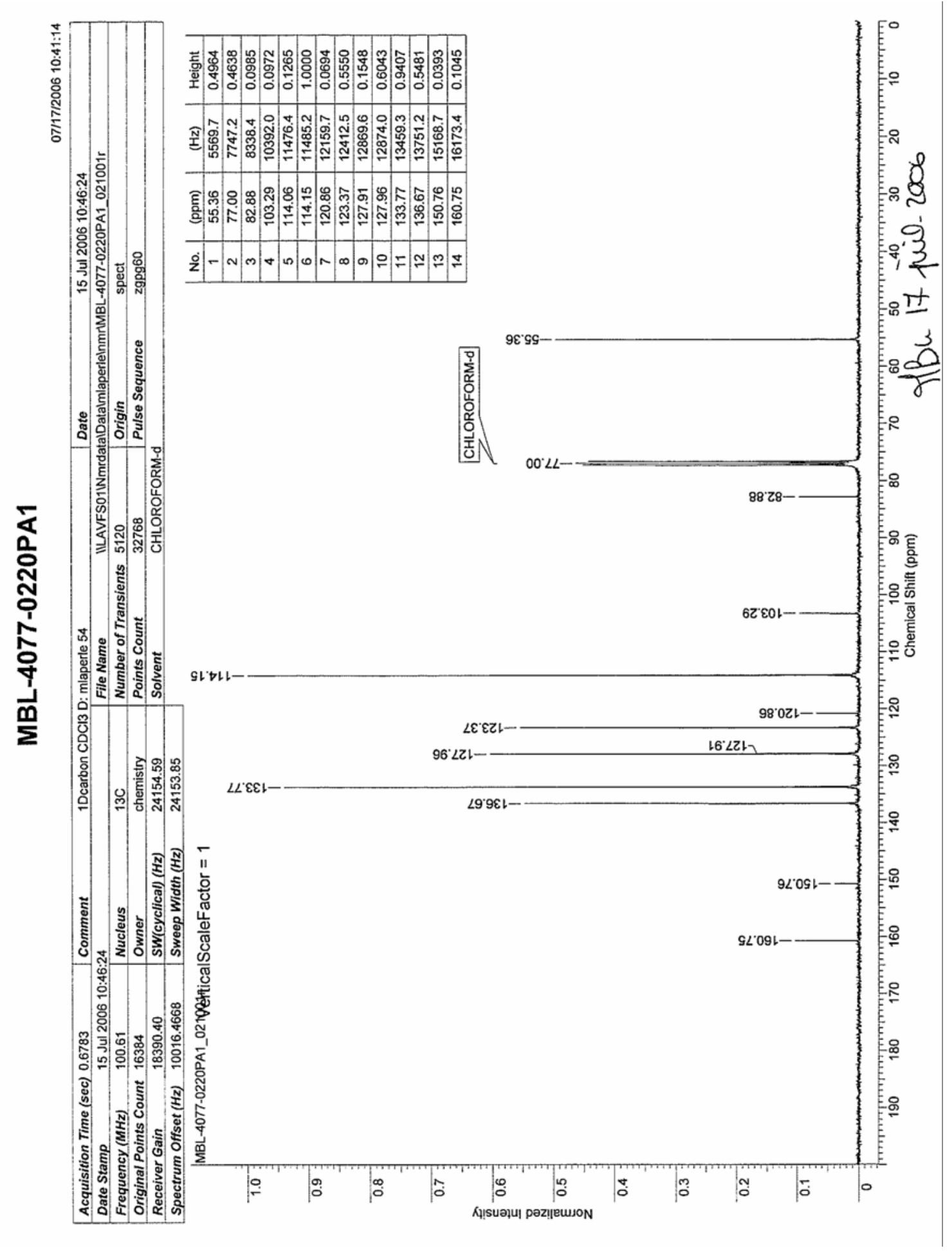




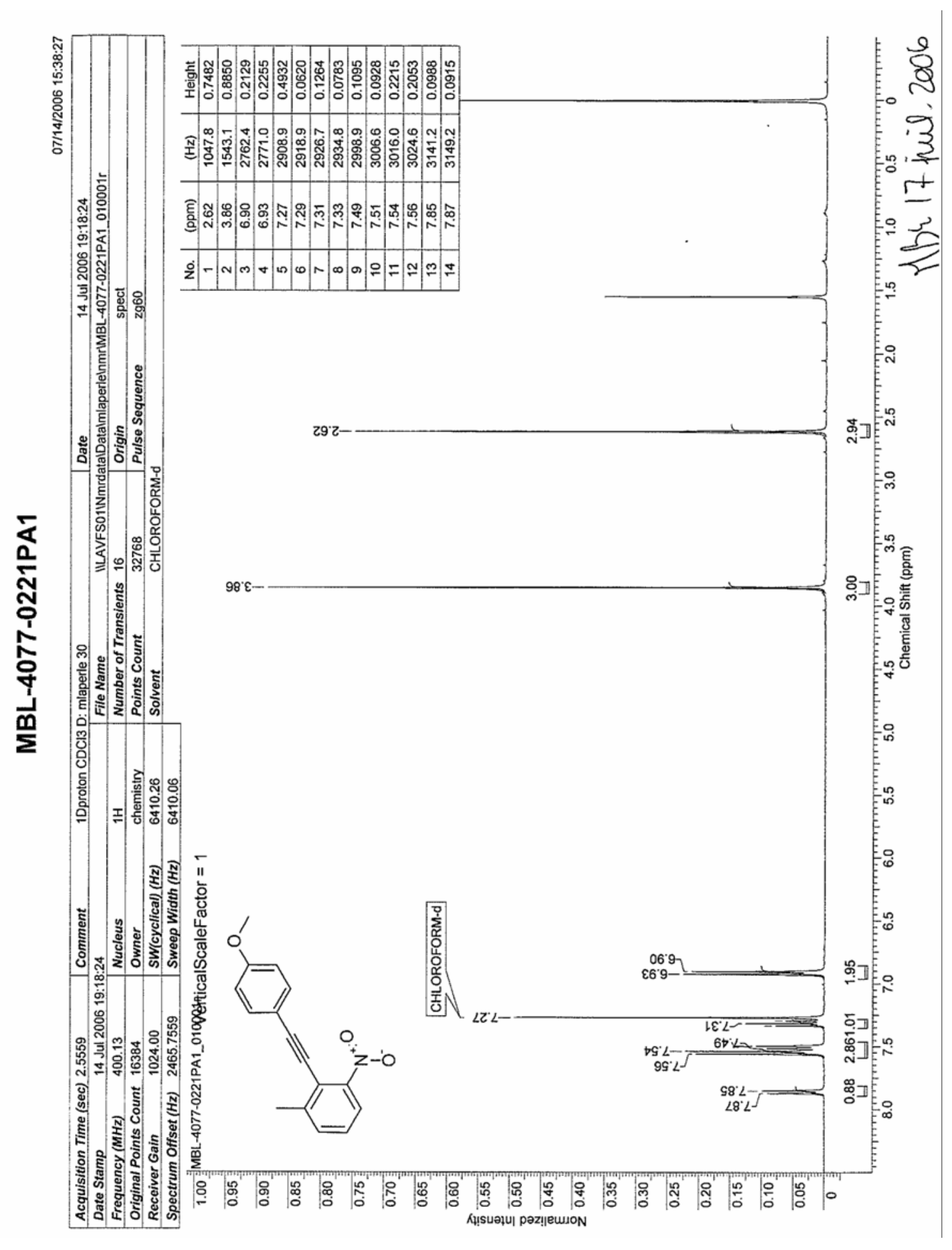




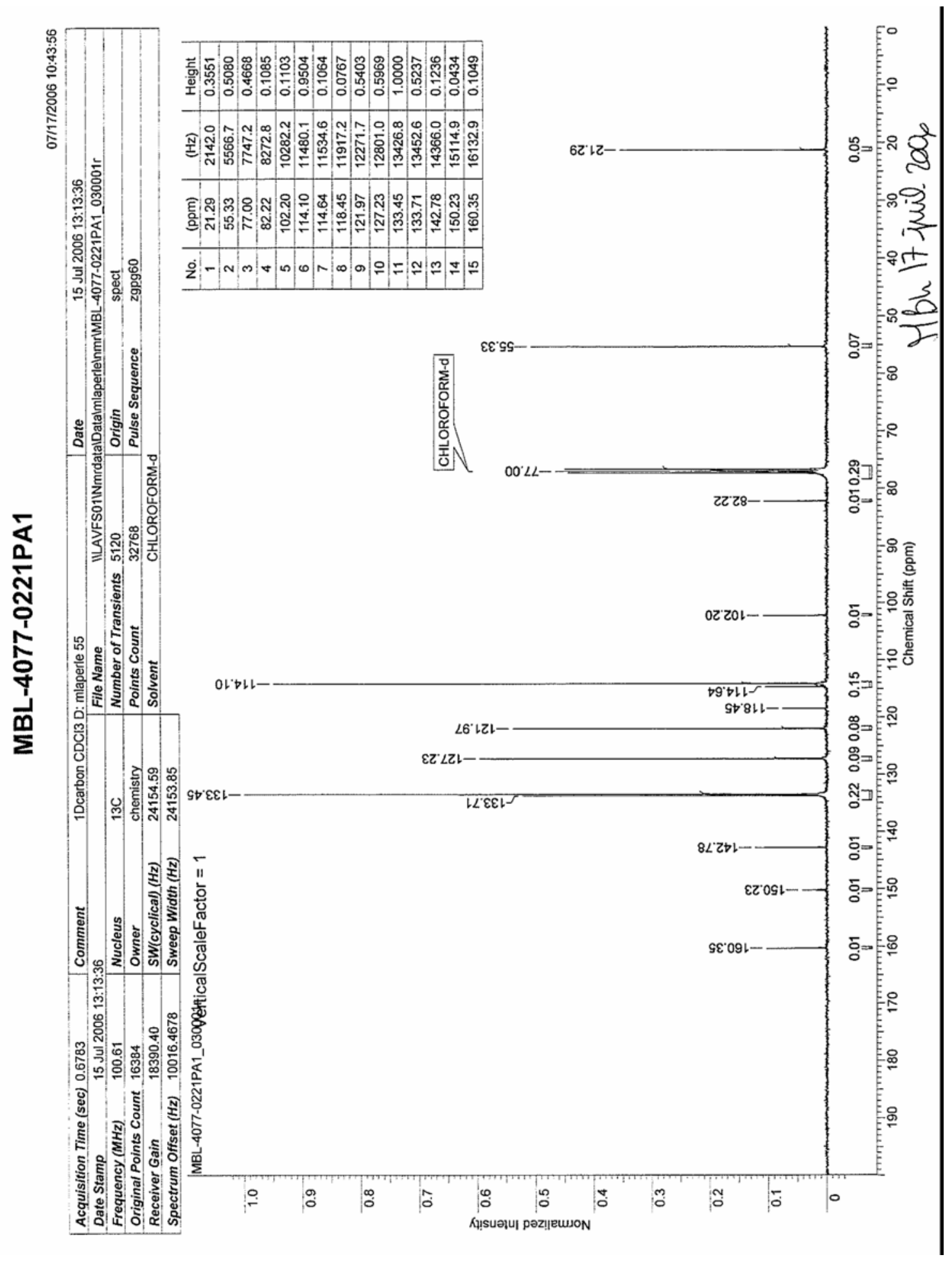




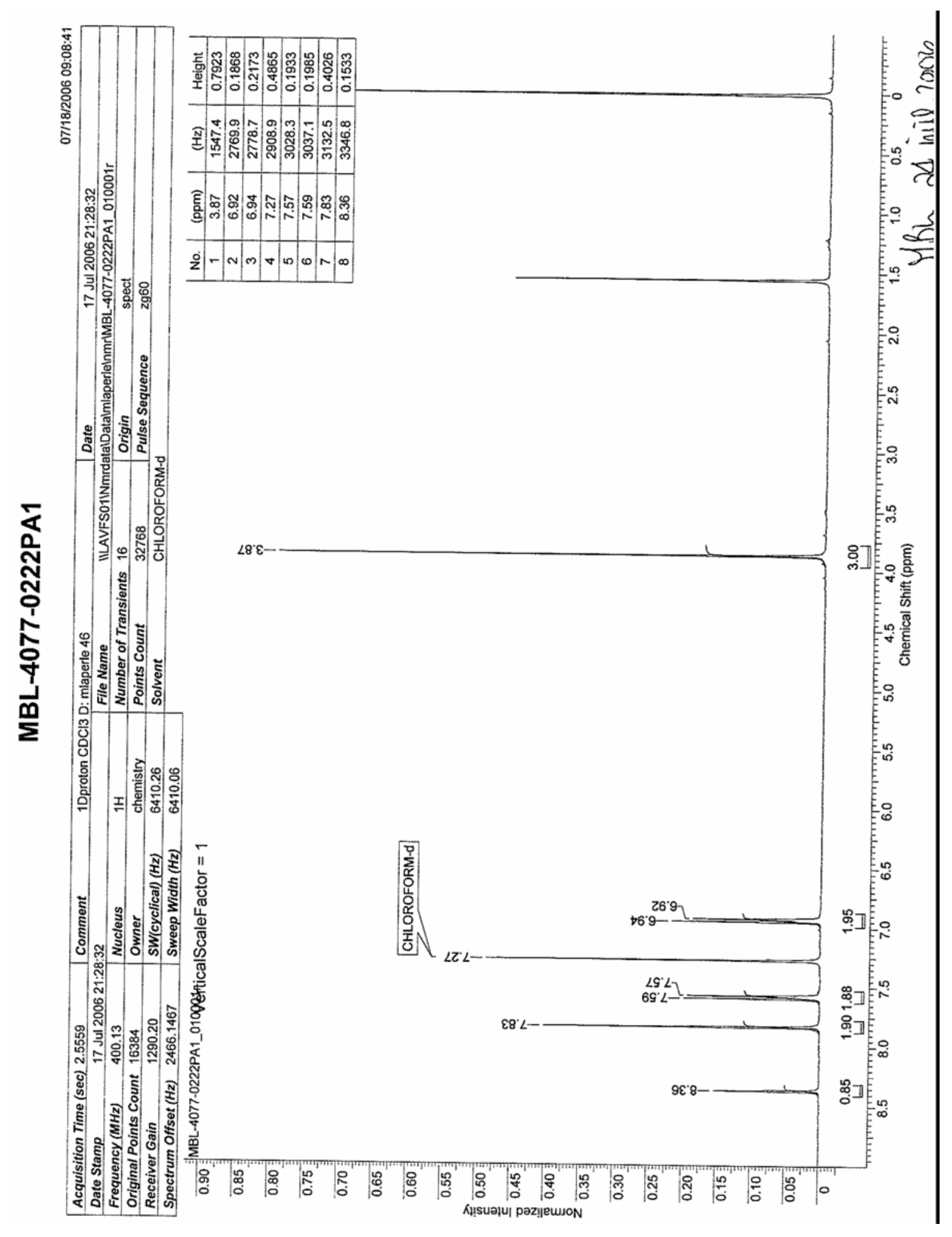




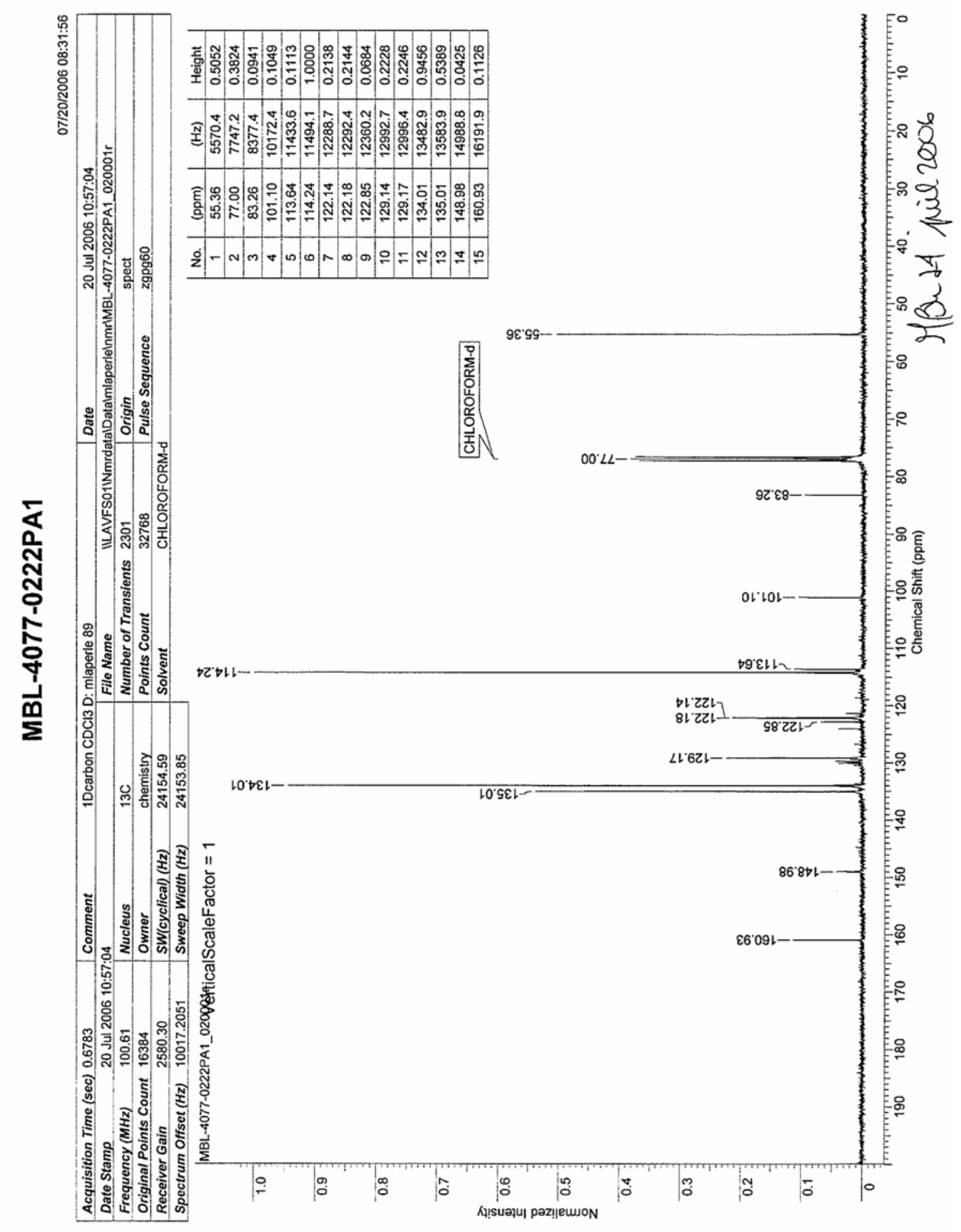




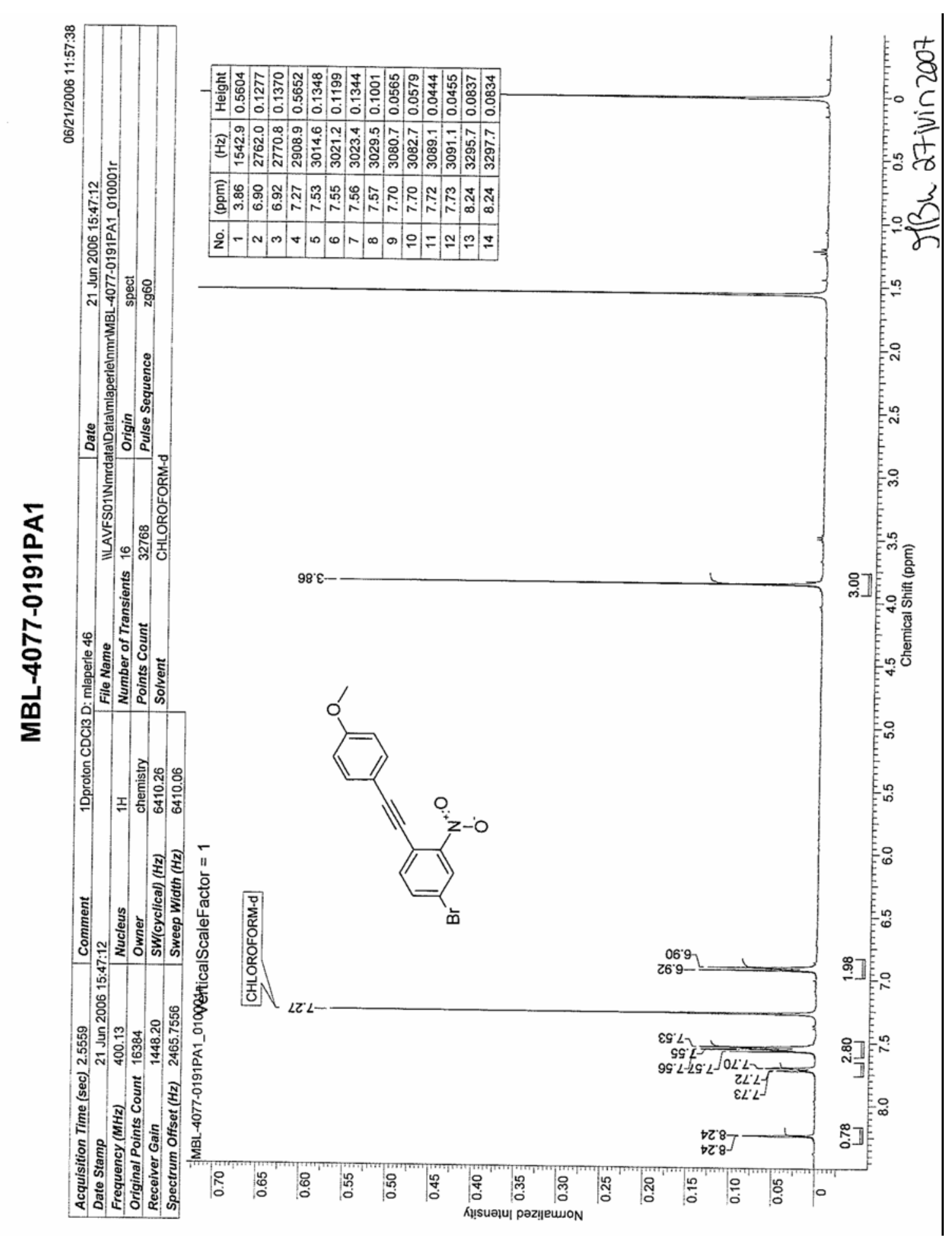




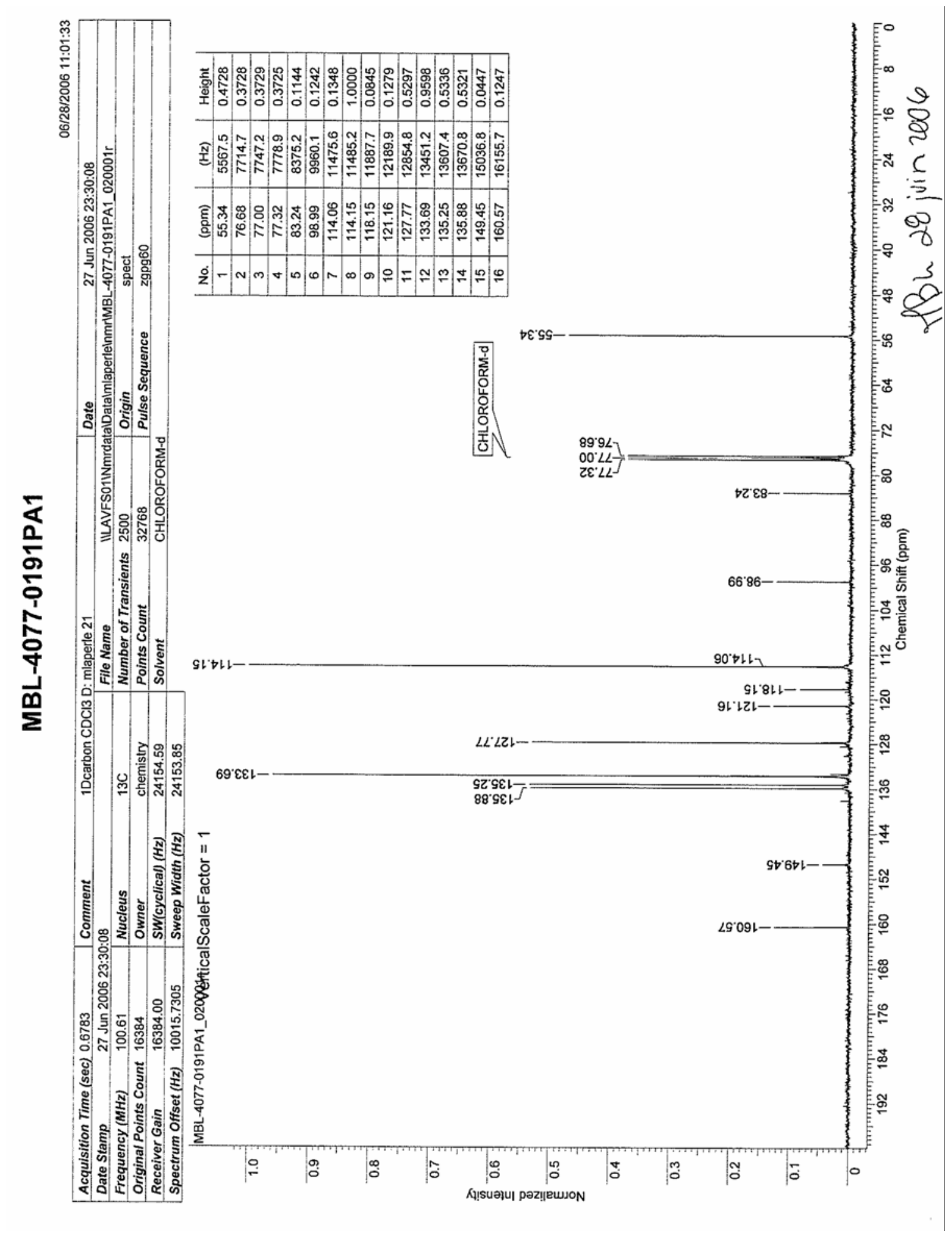




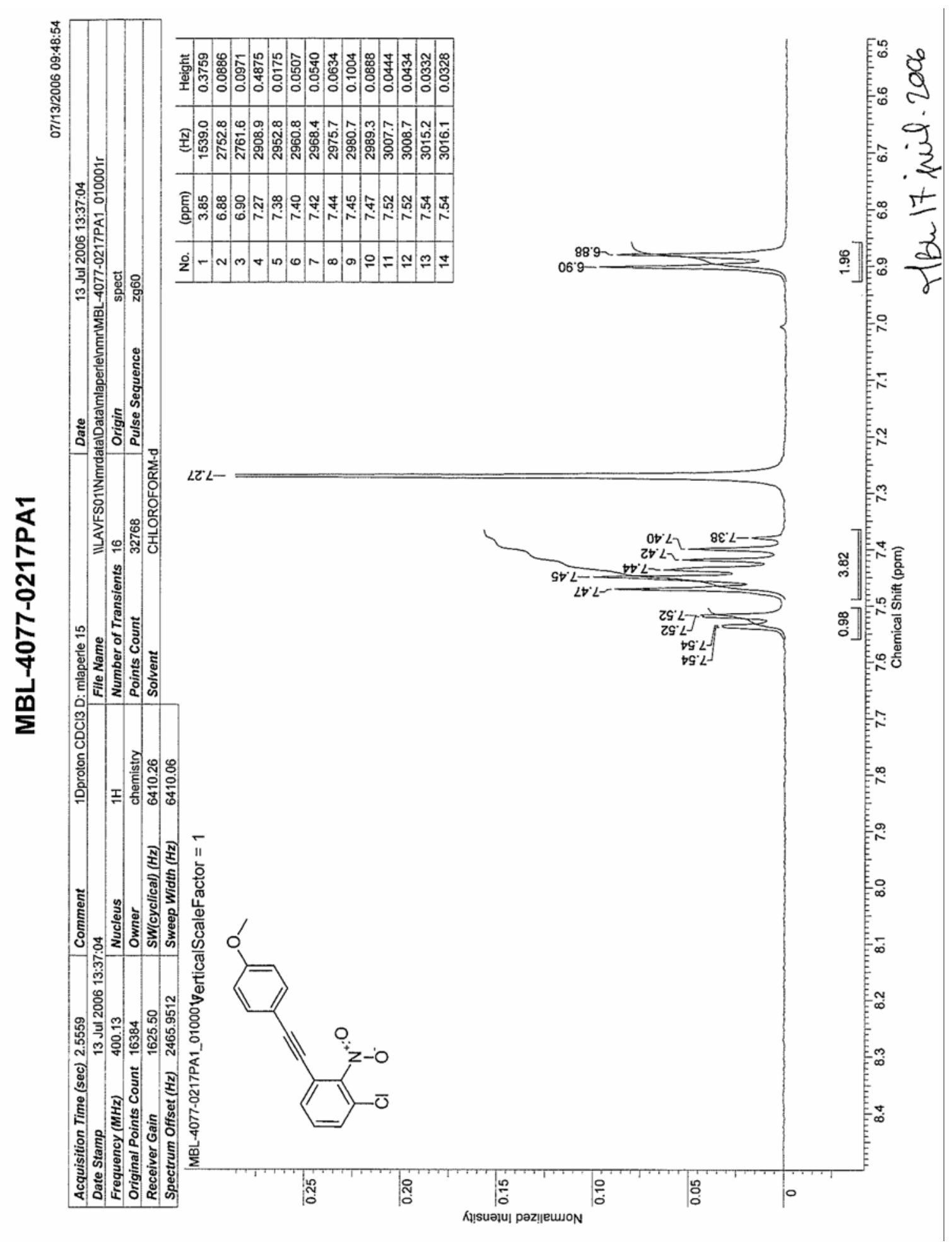




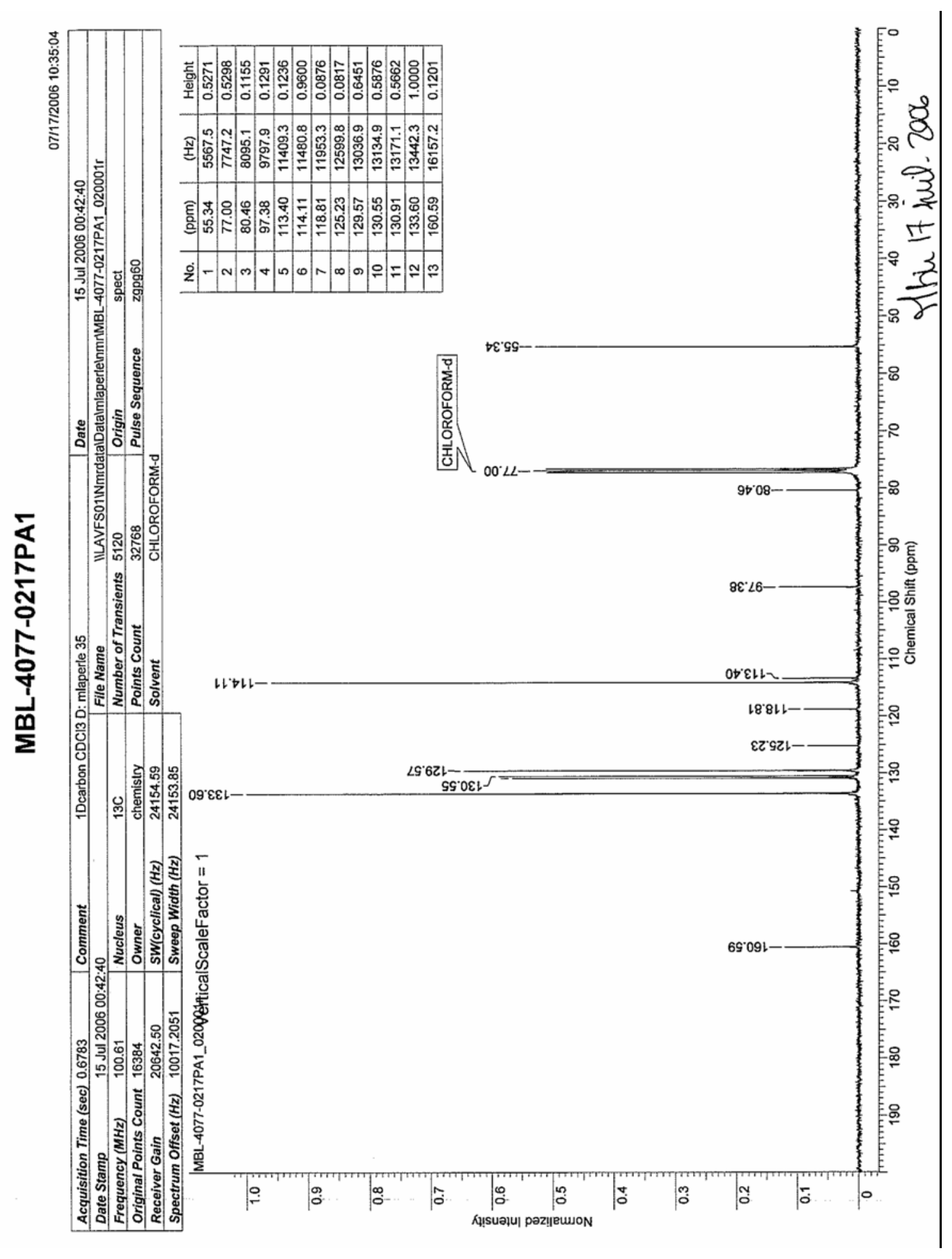

